\title{
STABILITY, FINITE-TIME STABILITY AND PASSIVITY CRITERIA FOR DISCRETE-TIME DELAYED NEURAL NETWORKS \\ UDC (681.037:004.032.26)
}

\section{Sreten Stojanović ${ }^{1}$, Miloš Stevanović1 Dragan Antić2, Milan Stojanović3}

\author{
${ }^{1}$ University of Niš, Faculty of Technology, Leskovac, Republic of Serbia \\ ${ }^{2}$ University of Niš, Faculty of Electronic Engineering, Niš, Republic of Serbia \\ ${ }^{3}$ University of Belgrade, School of Electrical Engineering, Belgrade, Republic of Serbia
}

\begin{abstract}
In this paper, we present the problem of stability, finite-time stability and passivity for discrete-time neural networks (DNNs) with variable delays. For the purposes of stability analysis, an augmented Lyapunov-Krasovskii functional (LKF) with single and double summation terms and several augmented vectors is proposed by decomposing the time-delay interval into two non-equidistant subintervals. Then, by using the Wirtinger-based inequality, reciprocally and extended reciprocally convex combination lemmas, tight estimations for sum terms in the forward difference of LKF are given. In order to relax the existing results, several zero equalities are introduced and stability criteria are proposed in terms of linear matrix inequalities (LMIs). The main objective for the finite-time stability and passivity analysis is how to effectively evaluate the finite-time passivity conditions for DNNs. To achieve this, some weighted summation inequalities are proposed for application to a finite-sum term appearing in the forward difference of $L K F$, which helps to ensure that the considered delayed DNN is passive. The derived passivity criteria are presented in terms of linear matrix inequalities. Some numerical examples are presented to illustrate the proposed methodology.
\end{abstract}

Key words: Stability, finite-time stability, finite-time passivity, neural networks, time delay, Lyapunov-Krasovskii functional

\section{INTRODUCTION}

During the past few decades, neural networks (NNs) have received great attention because of their wide applications in various fields such as image processing, signal

Received September 22, 2020

Corresponding author: Sreten B. Stojanović

University of Nis, Faculty of Technology, Bulevar oslobodjenja 124, 16000 Leskovac, Republic of Serbia

E-mail: sstojanovic@tf.ni.ac.rs 
processing, pattern recognition, associative memory, parallel computation, optimization, error diagnosis and so on [1,2]. One of the most important questions in theoretical analysis of NNs is dynamical behaviours of the NNs, such as their stability [3, 4], periodic oscillatory, and chaos. It is well known that a time delay is inherent in various systems, including NNs, owing to the finite speed of signal transmission and conversion rate of the processors. Delays in a system may cause oscillation and divergence and further degrade the performance [5-10]. Since most systems use a digital processor to acquire information from computers at discrete instants of time, it is essential to formulate discrete-time neural networks (DNNs) that are an analogue of continuous ones [11-18]. In order to improve results regarding this problem, various techniques have been applied to the delay-dependent category, such as augmented Lyapunov-Krasovskii (LK) functional [13, 19-22], free-weighting matrix method [18, 23], summation inequality method [16, 24-27], delay-partitioning method [5, 28, 29] and reciprocally convex approach [20, 30, 31].

The passivity is part of a broader general theory of dissipativeness, which postulates that the energy dissipated inside a dynamic system is less than the energy supplied from an external source and is often linked to stability problems. In particular, the main idea behind the passivity theory (PT) is that the passive property of a system can keep the system internally stable. PT has been established in various control problems, including $H_{\infty}$ control and strict output (or input)-positive realness, so it provides an effective tool for analyzing the dynamic behaviours of a nonlinear system, namely, stability [32], chaos control and synchronization [33], [34], signal processing [35], and complexity [36]. Passivity has been analyzed for various systems, specifically NNs and chaotic, linear, and switched systems [37-42].

Generally, existing results on the passivity problem for DNNs have been from studies considering an infinite-time interval. However, the dynamic properties of the system have been studied over a fixed short time in many practical applications, such as biochemical reaction systems, communication network systems, and other engineering systems. The finite-time stability (FTS) approach was introduced by Dorato in [43]. A very few FTS problems have been studied for discrete-time cases in the literature [44-47]. In addition, Mathiyalagan et al. [48] examined the robust finite-time passivity (FTP) problem of DNNs with time delay by using the concept of finite-time boundedness. However, this approach might have produced conservative results, which has motivated authors in [16] to improve the FTP criteria of DNNs using new weighted summation inequalities.

After the computational complexity became one of the crucial aspects of a research in the area of the system stability, the direct bounding method based on summation inequalities once again becomes the most popular method [17, 19, 49, 50]. Very recently, various types of the Wirtinger-based summation inequalities, tighter than the Jensenbased summation inequality, have been proposed for discrete-time linear time-delay systems $[25,51,52]$ and have also been used for the study of the discrete-time DNNs [39]. As one of useful methods to deal with the stability of delayed systems, the reciprocally convex approach was developed in [30] and has been extensively used to study the dynamical behaviours of time-delay systems [32, 53-55].

The goal of this paper is to present the main authors' results in the stability [56], finite-time stability and finite-time passivity analysis [16] of discrete-time neural networks with interval time-varying delay. 
In order to reduce the conservatism of proposed stability criteria, delay-decomposition method (DDM) is used in [56]. DDM is based on a discrete LKF with a free parameter $\alpha$ that divides the summation discrete interval $\left[k-h_{2}, k-h_{1}\right]$ into two asymmetric discrete subintervals $\left[k-h_{2}, k-\alpha-1\right]$ and $\left[k-\alpha, k-h_{1}\right]$. In this way, a greater degree of freedom is enabled in estimating the stability of the DDN, which leads to a smaller conservative stability criterion. Further, the convenient summations inequalities, extended reciprocally convex combination lemma and zero equalities (ZEs) have been used for calculation difference of KLF. As a result of applying the mentioned techniques, some less conservative results are derived in [56]. As a result of applying the mentioned techniques, it is shown that the derived results are less conservative then the existing ones [24, 31, 57, 58].

Using the concepts of FTS and FTP, the researchers in [16] are focused on developing a new method to analyse the FTP of delayed DNNs. To reach this goal, a new definition of EP for DNNs is introduced and new finite-sum inequalities with exponential functions are proposed. A suitable Lyapunov- Krasovskii functional (LKF) with single, double, and triple sums is proposed. To obtain less conservative results, the proposed weighted summation inequalities and a reciprocal convex combination approach are used in [16].

Finally, the numerical examples are presented to illustrate the effectiveness of the proposed results and their improvement over the existing literature.

Section 2 presents the problem formulation and mathematical preliminaries. In Section 3, we present the stability, finite-time stability and finite-time passivity of DDNs. Section 4 presents a numerical example to validate the proposed results.

Notations. Throughout the paper, $\square^{+}$denotes the set of positive integers, $\mathfrak{R}^{n}$ denotes the $n$-dimensional Euclidean space and $\mathfrak{R}^{n \times m}$ the set of all $n \times m$ real matrices. For the positive integers $a$ and $b(b>a), \square[a, b]$ denotes the set of all positive integers $z$ satisfying $a \leq z \leq b . I_{n}$ and $0_{n \times m}$ denote the $n \times n$ identity matrix and $n \times m$ zero matrix, respectively. $X^{T}$ denotes the matrix transpose of $X$ and * represent the elements below the main diagonal of a symmetric matrix. $\operatorname{diag}\{a, b, \ldots, z\}$ denotes the block-diagonal matrix with elements $a, b, \ldots, z$ in the diagonal entries and $\operatorname{Sym}\{X\}=X+X^{T}$. For any symmetric matrix $X \in \mathfrak{R}^{n \times n}$ the notation $X>0 \quad(X \geq 0)$ means that $X$ is a positive definite (positive semi-definite) matrix and $X<0 \quad(X \leq 0)$ means that $X$ is a negative definite (negative semi-definite) matrix. For the matrices $A_{i} \in \mathfrak{R}^{n \times m}, i=1,2, \cdots, l$, $\operatorname{Col}\left\{A_{1}, A_{2}, \cdots, A_{l}\right\}$ denotes the column block matrix $\left[A_{1}^{T} A_{2}^{T} \cdots A_{l}^{T}\right]^{T}$. Matrices, if their dimensions are not explicitly stated, are assumed to be compatible for algebraic operations.

\section{PRELIMINARIES}

Consider the discrete-time neural networks (DNNs) with an interval time-varying delay of the form $[16,56]$ :

$$
\begin{aligned}
& x(k+1)=C x(k)+A f(x(k))+A_{d} f(x(k-h(k)))+u(t), \quad k \geq 0 \\
& y(k)=f(x(k)), \\
& x(j)=\phi(j), \quad j \in\left\{-h_{2},-h_{2}+1, \cdots,-1,0\right\}
\end{aligned}
$$

where $k \in \square^{+}, x(k) \in \mathfrak{R}^{n}$ is the state vector, $y(k)$ is the output vector, $u(k)$ is the exogenous disturbance input vector, $C, A, A_{d} \in \mathfrak{R}^{n \times n}$ are the known real constant matrices, 
$f(\cdot)=\left[f_{1}(\cdot) f_{2}(\cdot) \cdots f_{n}(\cdot)\right]^{T}$ denotes nonlinear activation functions with $f_{i}(0)=0$, and $h(k)$ is the time-varying delay satisfying

$$
0<h_{1} \leq h(k) \leq h_{2},
$$

where $h_{1}$ and $h_{2}$ are the known positive constants. In addition, $\phi(j)$ denotes a vectorvalued initial function that satisfies

$$
\sup _{j \in\left\{-h_{2},-h_{2}+1, \cdots,-1\right\}}(\phi(j+1)-\phi(j))^{T}(\phi(j+1)-\phi(j)) \leq \delta
$$

where $\delta$ is a positive constant.

In this paper, we make the following assumption.

Assumption 1. [48] For any $s_{1}, s_{2} \in \mathfrak{R}, s_{1} \neq s_{2}$, the continuous and bounded activation functions $f_{i}(\cdot)$ satisfy

$$
k_{i}^{-} \leq \frac{f_{i}\left(\mathrm{~s}_{1}\right)-f_{i}\left(\mathrm{~s}_{2}\right)}{s_{1}-s_{2}} \leq k_{i}^{+}, \quad i=1,2, \cdots, n
$$

where $k_{i}^{-}$and $k_{i}^{+}$are known constants. For $s_{2}=0$ we have $f(0)=0$ and

$$
k_{i}^{-} \leq \frac{f_{i}\left(s_{1}\right)}{s_{1}} \leq k_{i}^{+}, \quad i=1,2, \cdots, n
$$

for all $s_{1} \neq 0$.

The following lemmas will be used in the sequel to establish the main results.

Lemma 1. (Jensen's inequality [59]) For any positive definite symmetric matrix $R \in \mathfrak{R}^{n \times n}$, two positive integers $a$ and $b>a$, the sum term $\sum_{j=a}^{b-1} x^{T}(j) R x(j)$ is estimated as

$$
(b-a) \sum_{j=a}^{b-1} x^{T}(j) R x(j) \geq \sum_{j=a}^{b-1} x^{T}(j) R \sum_{j=a}^{b-1} x(j)
$$

Lemma 2. (Wirtinger-based inequality [25])

For a given positive definite symmetric matrix $R$, two positive integers $a$ and $b>a$, any sequence of discrete-time variable $x: \square[a, b] \rightarrow \Re^{n}$, the following inequality holds

$$
(b-a) \sum_{i=a}^{b-1} \eta^{T}(i) R \eta(i) \geq\left[\begin{array}{l}
\theta_{1} \\
\theta_{2}
\end{array}\right]^{T}\left[\begin{array}{cc}
R & 0 \\
* & 3 \rho(a, b) R
\end{array}\right]\left[\begin{array}{l}
\theta_{1} \\
\theta_{2}
\end{array}\right] \geq\left[\begin{array}{c}
\theta_{1} \\
\theta_{2}
\end{array}\right]^{T}\left[\begin{array}{cc}
R & 0 \\
* & 3 R
\end{array}\right]\left[\begin{array}{l}
\theta_{1} \\
\theta_{2}
\end{array}\right]
$$

where

$$
\begin{aligned}
& \eta(k)=x(k+1)-x(k), \quad \theta_{1}=x(b)-x(a), \\
& \theta_{2}=x(b)+x(a)-\frac{2}{b-a+1} \sum_{i=a}^{b} x(i) \\
& \rho(a, b)= \begin{cases}(b-a+1) /(b-a-1), & b-a \neq 1 \\
1, & b-a=1\end{cases}
\end{aligned}
$$


The reciprocally convex combination lemma (RCCL) plays an important role in the estimation of the forward difference of LKF.

Lemma 3. (Reciprocally convex combination lemma [30, 31, 60]) For a real scalar $\delta \in(0,1)$, a symmetric matrix $R>0$, and any matrix $S$ satisfying

$$
\left[\begin{array}{ll}
R & S \\
* & R
\end{array}\right] \geq 0
$$

the following inequality holds

$$
\left[\begin{array}{cc}
\frac{1}{\delta} R & 0 \\
* & \frac{1}{1-\delta} R
\end{array}\right] \geq\left[\begin{array}{cc}
R & S \\
* & R
\end{array}\right]
$$

An extended reciprocally convex combination lemma (ERCCL), which estimate the sum terms in the forward difference of LKF tightly than RCCL is presented as follows.

Lemma 4. (Extended reciprocally convex combination lemma [31, 57]) For a real scalar $\delta \in(0,1)$, a symmetric matrix $R>0$, and any matrix $S$, the following inequality holds

$$
\left[\begin{array}{cc}
\frac{1}{\delta} R & 0 \\
* & \frac{1}{1-\delta} R
\end{array}\right] \geq\left[\begin{array}{cc}
R+(1-\delta) T_{1} & S \\
* & R+\delta T_{2}
\end{array}\right]
$$

where $T_{1}=R-S R^{-1} S^{T}$ and $T_{2}=R-S^{T} R^{-1} S$.

Remark 1. In reference [20], the specific reciprocally convex inequality is proposed. For comparison, we rewrite the result of Theorem 1 in [20] in a block form as

$$
\left[\begin{array}{cc}
\frac{1}{\delta} R_{1} & 0 \\
* & \frac{1}{1-\delta} R_{2}
\end{array}\right] \geq\left[\begin{array}{cc}
R_{1}+(1-\delta) T_{1} & \delta S_{1}+(1-\delta) S_{2} \\
* & R_{2}+\delta T_{2}
\end{array}\right]
$$

where $T_{1}=R_{1}-Y_{1} R_{2}^{-1} Y_{1}^{T}$ and $T_{2}=R_{2}-Y_{2}^{T} R_{1}^{-1} Y_{2}$. If taking $R_{1}=R_{2}=R$ and $S_{1}=S_{2}=S$, inequality (12) immediately reduces to (11), which means that ERCCL (Lemmas 4) is a special case of Theorem 1 in [20].

Lemma 5. ([57]) For a positive definite symmetric matrix $R$ matrices $\Upsilon$ and $\Xi$, the following statements are equivalent

(i) $\Xi-\Upsilon^{T} R \Upsilon<0$

(ii) There exists a matrix $\Psi$ with appropriate dimension such that

$$
\left[\begin{array}{cc}
\Xi+\operatorname{Sym}\left\{\Upsilon^{T} \Psi\right\} & \Psi^{T} \\
* & -R
\end{array}\right]<0
$$

To study the finite-time stability of the DNN (1), we introduce the following definitions.

Definition 1. [61] The DNN (1) with time varying delay is said to be finite-time stable with respect to $(\alpha, \beta, N)$, where $0 \leq \alpha<\beta$, if

$$
\sup _{j \in\left\{-h_{2},-h_{2}+1, \cdots, 0\right\}} x^{T}(j) x(j) \leq \alpha \Rightarrow x^{T}(k) x(k)<\beta, \quad \forall k \in\{1,2, \cdots, N\}
$$


Definition 2. [16] With respect to $(\alpha, \beta, N, \mu)$, where $0 \leq \alpha<\beta, \gamma \geq 1$ and $\mu$ is a positive scalar, DNN (1) with time-varying delay is said to be finite-time passive if it is FTS with respect to $(\alpha, \beta, N)$, and under the zero initial condition, output $y(k)$ satisfies

$$
2 \sum_{j=0}^{k-1} \gamma^{-j} y^{T}(j) u(j) \geq-\mu \sum_{j=0}^{k-1} u^{T}(j) u(j), \quad \forall k \in\{1,2, \cdots, N\}
$$

Remark 2. Since the system output $y(j)$ in (15) is scaled by the exponential function $\gamma^{-j}$, then the exponential behaviour of discrete systems is embedded in the inequality (15). Therefore, we define the condition (15) as exponential passivity (EP) for discrete-time systems. This EP is analogous to EP for continuous-time systems which are considered in the existing literature. In the case $\gamma=1$, the inequality (15) becomes a well-known condition for the passivity of discrete-time systems

$$
2 \sum_{j=0}^{k-1} y^{T}(j) u(j) \geq-\mu \sum_{j=0}^{k-1} u^{T}(j) u(j)
$$

Based on Definition 2, FTP is expressed as a combination of two properties: the exponential passivity and the finite-time stability.

Before deriving the main results, we introduce three weighted summation inequalities, which play a significant role in obtaining new criteria.

Lemma 6. [16] For given integers $h_{1}, h_{2}, 0 \leq h_{1}<h_{2}$, and $\gamma>0$, a vector function $\omega(k)$, $k \in Z^{+}$, and symmetric matrix $R>0$, the following inequalities hold:

$$
\begin{aligned}
& \pi \sum_{j=k-h_{2}}^{k-h_{1}-1} \gamma^{k-j} \omega^{T}(j) R \omega(j) \geq\left(\sum_{j=k-h_{2}}^{k-h_{1}-1} \omega(j)\right)^{T} R\left(\sum_{j=k-h_{2}}^{k-h_{1}-1} \omega(j)\right) \\
& \kappa \sum_{i=-h_{2}} \sum_{j=k+i}^{k-1} \gamma^{k-j} \omega^{T}(j) R \omega(j) \geq\left(\sum_{i=-h_{2}}^{-h_{1}-1} \sum_{j=k+i}^{k-1} \omega(j)\right)^{T} R\left(\sum_{i=-h_{2}}^{-h_{1}-1} \sum_{j=k+i}^{k-1} \omega(j)\right) \\
& \vartheta \sum_{i=k-h_{2}}^{k-h_{1}-1} \sum_{j=i}^{k-h_{1}-1} \gamma^{k-j} \omega^{T}(j) R \omega(j) \geq\left(\sum_{i=k-h_{2}}^{k-h_{1}-1} \sum_{j=i}^{k-h_{1}-1} \omega(j)\right)^{T} R\left(\sum_{i=k-h_{2}}^{k-h_{1}-1} \sum_{j=i}^{k-h_{1}-1} \omega(j)\right)
\end{aligned}
$$

where

$$
\pi=\sum_{j=k-h_{2}}^{k-h_{1}-1} \gamma^{-(k-j)}, \quad \kappa=\sum_{i=-h_{2}}^{-h_{1}-1} \sum_{j=k+i}^{k-1} \gamma^{-(k-j)}, \quad \vartheta=\sum_{i=k-h_{2}}^{k-h_{1}-1} \sum_{j=i}^{k-h_{1}-1} \gamma^{-(k-j)}
$$

Proof. Let $X=\left[\begin{array}{cc}R^{1 / 2} Y & R^{-1 / 2} \\ 0 & 0\end{array}\right]$ with $Y \in \mathfrak{R}^{n}, 0<R \in \mathfrak{R}^{n \times n}$. Then,

$$
X^{T} X=\left[\begin{array}{cc}
Y^{T} R^{1 / 2} & 0 \\
R^{-1 / 2} & 0
\end{array}\right]\left[\begin{array}{cc}
R^{1 / 2} Y & R^{-1 / 2} \\
0 & 0
\end{array}\right]=\left[\begin{array}{cc}
Y^{T} R Y & Y^{T} \\
Y & R^{-1}
\end{array}\right] \geq 0
$$


Replacing $Y$ and $R$ in (21) by $\omega(j)$ and $\gamma^{k-j} R$, respectively and summing from $k-h_{2}$ to $k-h_{1}-1$ gives

$$
\sum_{j=k-h_{2}}^{k-h_{1}-1}\left[\begin{array}{cc}
\omega^{T}(j) \gamma^{k-j} R \omega(j) & \omega^{T}(j) \\
\omega(j) & \gamma^{-(k-j)} R^{-1}
\end{array}\right] \geq 0
$$

Through the Schur complement, we obtain

$$
\begin{aligned}
\sum_{j=k-h_{2}}^{k-h_{1}-1} \gamma^{k-j} \omega^{T}(j) R \omega(j) & \geq\left(\sum_{j=k-h_{2}}^{k-h_{1}-1} \omega(j)\right)^{T}\left(\sum_{j=k-h_{2}}^{k-h_{1}-1} \gamma^{-(k-j)}\right)^{-1} R\left(\sum_{j=k-h_{2}}^{k-h_{1}-1} \omega(j)\right) \\
& =\pi^{-1}\left(\sum_{j=k-h_{2}}^{k-h_{1}-1} \omega(j)\right)^{T} R\left(\sum_{j=k-h_{2}}^{k-h_{1}-1} \omega(j)\right)
\end{aligned}
$$

where $\pi=\sum_{j=k-h_{2}}^{k-h_{1}-1} \gamma^{-(k-j)}$, which is equivalently represented by $\pi$ in (20). We then obtain (17). Using a similar method, we obtain

$$
\sum_{i=-h_{2}}^{-h_{1}-1} \sum_{j=k+i}^{k-1}\left[\begin{array}{cc}
\omega^{T}(j) \gamma^{k-j} R \omega(j) & \omega^{T}(j) \\
\omega(j) & \gamma^{-(k-j)} R^{-1}
\end{array}\right] \geq 0
$$

which leads to

$$
\begin{aligned}
\sum_{i=-h_{2}}^{-h_{1}-1} \sum_{j=k+i}^{k-1} \gamma^{k-j} \omega^{T}(j) R \omega(j) & \leq\left(\sum_{i=-h_{2}}^{-h_{1}-1} \sum_{j=k+i}^{k-1} \omega(j)\right)^{T}\left(\sum_{i=-h_{2}}^{-h_{1}-1} \sum_{j=k+i}^{k-1} \gamma^{-(k-j)}\right)^{-1} R\left(\sum_{i=-h_{2}}^{-h_{1}-1} \sum_{j=k+i}^{k-1} \omega(j)\right) \\
& =\kappa^{-1}\left(\sum_{i=-h_{2}}^{-h_{1}-1} \sum_{j=k+i}^{k-1} \omega(j)\right)^{T} R\left(\sum_{i=-h_{2}}^{-h_{1}-1} \sum_{j=k+i}^{k-1} \omega(j)\right)
\end{aligned}
$$

where $\kappa=\sum_{i=-h_{2}}^{-h_{1}-1} \sum_{j=k+i}^{k-1} \gamma^{-(k-j)}$. Thus, we obtain (18). A similar proof can be derived for the inequality (19).

Remark 3. Jensen's inequities for the single and double summation represent the special cases $(\gamma=1)$ of inequalities (17)-(19).

Lemma 7. [30] For given positive integers $n$ and $m$, a scalar $\alpha$ in the interval $(0,1)$, a given $n \times m$ matrix $R>0$, and two matrices $W_{1}$ and $W_{2}$ in $\mathfrak{R}^{n \times m}$, for all vectors $\xi$ in $\mathfrak{R}^{m}$ let us define the function $\theta(\alpha, R)$ given by

$$
\theta(\alpha, R)=\frac{1}{\alpha} \xi^{T} W_{1}^{T} R W_{1} \xi+\frac{1}{1-\alpha} \xi^{T} W_{2}^{T} R W_{2} \xi
$$


Then, if there exists a matrix $X$ in $\mathfrak{R}^{n \times n}$ such that $\left[\begin{array}{cc}R & X \\ * & R\end{array}\right]>0$, the following inequality holds:

$$
\min _{\alpha \in(0,1)} \theta(\alpha, R) \geq\left[\begin{array}{l}
W_{1} \xi \\
W_{2} \xi
\end{array}\right]^{T}\left[\begin{array}{ll}
R & X \\
* & R
\end{array}\right]\left[\begin{array}{c}
W_{1} \xi \\
W_{2} \xi
\end{array}\right]
$$

Lemma 8. [53] For symmetric matrices of appropriate dimensions $R>0, \Omega$, and a matrix $\Gamma$, the following two statements are equivalent:

(1) $\Omega-\Gamma R \Gamma^{T}<0$.

(2) There exist a matrix of the appropriate dimensions $\Pi$ such that

$$
\left[\begin{array}{cc}
\Omega+\Gamma \Pi^{T}+\Pi \Gamma^{T} & \Pi \\
\Pi^{T} & -R
\end{array}\right]<0
$$

\section{MAIN RESULTS}

\subsection{Stability of DNNs with time-varying delay}

In this section, we present a criterion on the asymptotical stability for the discrete-time neural networks with interval time-varying delay (1) that was proposed in [56]. The following notations are introduced for later use:

$$
\begin{aligned}
& \xi(k)=\left[\begin{array}{lllllll}
x^{T}(k) & x^{T}\left(k-h_{1}\right) & x^{T}(k-h(k)) & x^{T}\left(k-h_{2}\right) & x^{T}(k-\alpha) & v_{1}^{T}(k) & v_{2}^{T}(k)
\end{array}\right. \\
& \left.v_{3}^{T}(k) v_{4}^{T}(k) f^{T}(x(k)) f^{T}(x(k-h(k)))\right]^{T} \\
& \zeta_{1}(k)=\left[x^{T}(k) \sum_{j=k-h_{1}}^{k-1} x^{T}(j) \sum_{j=k-h_{2}}^{k-h_{1}-1} x^{T}(j) \sum_{j=k-\alpha}^{k-h_{1}-1} x^{T}(j)\right]^{T}, \eta(k)=x(k+1)-x(k) \\
& \zeta_{2}(k)=\left[\begin{array}{lll}
x^{T}(k) & f^{T}(x(k))
\end{array}\right]^{T}, \quad \xi_{3}(k)=\left[\begin{array}{ll}
x^{T}(k) & \eta^{T}(x(k))
\end{array}\right]^{T} \\
& v_{1}(k)=\sum_{j=k-h_{1}}^{k} \frac{x(j)}{h_{1}+1}, \quad v_{2}(k)=\sum_{j=k-h(k)}^{k-h_{1}} \frac{x(j)}{h(k)-h_{1}+1} \\
& v_{3}(k)=\sum_{j=k-h_{2}}^{k-h(k)} \frac{x(j)}{h_{2}-h(k)+1}, \quad v_{4}(k)=\sum_{j=k-\alpha}^{k-h_{1}} \frac{x(j)}{\alpha-h_{1}+1} \\
& \omega_{1}=\left(e_{1}-e_{2}\right) \xi(k), \quad \omega_{2}=\left(e_{1}+e_{2}-2 e_{6}\right) \xi(k) \\
& \omega_{3}=\left(e_{2}-e_{5}\right) \xi(k), \quad \omega_{4}=\left(e_{2}+e_{5}-2 e_{9}\right) \xi(k) \\
& e_{i}=\left[0_{n \times(i-1) n}, I_{n}, 0_{n \times(11-i) n}\right], i=1,2, \cdots, 11 \\
& e_{s}=C e_{1}+A e_{10}+A_{d} e_{11}, \quad \rho(a, b)=\left\{\begin{array}{cc}
\frac{b-a+1}{b-a-1}, & b-a>1 \\
1, & b-a=1
\end{array},\right.
\end{aligned}
$$




$$
\begin{aligned}
& \delta_{h}=\frac{h(k)-h_{1}}{h_{12}}, \quad h_{12}=h_{2}-h_{1} \\
& \Sigma(h(k), \alpha)=\Sigma_{11}+\Sigma_{12}(h(k), \alpha)+\Sigma_{2}+\Sigma_{3}+\Sigma_{41}-\Sigma_{42} \\
& -\Sigma_{43}(h(k))+\Sigma_{51}(\alpha)-\Sigma_{52}(\alpha)-\Sigma_{S} \\
& \Sigma_{11}=\Pi_{1}^{T} P \Pi_{1}-\Pi_{2}^{T} P \Pi_{2}, \Sigma_{12}(h(k), \alpha)=\operatorname{Sym}\left\{\left(\Pi_{1}-\Pi_{2}\right)^{T} P \Pi(h(k), \alpha)\right\} \\
& \Pi_{1}=\left[\begin{array}{c}
e_{s} \\
\left(h_{1}+1\right) e_{6}-e_{2} \\
-e_{3}-e_{4} \\
-e_{5}
\end{array}\right], \quad \Pi_{2}=\left[\begin{array}{c}
e_{1} \\
\left(h_{1}+1\right) e_{6}-e_{1} \\
-e_{2}-e_{3} \\
-e_{2}
\end{array}\right] \\
& \Pi(h(k), \alpha)=\left[\begin{array}{c}
0 \\
0 \\
\left(h(k)-h_{1}+1\right) e_{7}+\left(h_{2}-h(k)+1\right) e_{8} \\
\left(\alpha-h_{1}+1\right) e_{9}
\end{array}\right] \\
& \Sigma_{2}=e_{1}^{T} Q_{1} e_{1}+e_{2}^{T}\left(Q_{3}-Q_{1}\right) e_{2}+e_{5}^{T}\left(Q_{2}-Q_{3}\right) e_{5}-e_{4}^{T} Q_{2} e_{4} \\
& \Sigma_{3}=\left[\begin{array}{c}
e_{1} \\
e_{10}
\end{array}\right]^{T}\left(h_{12}+1\right) R\left[\begin{array}{c}
e_{1} \\
e_{10}
\end{array}\right]-\left[\begin{array}{c}
e_{3} \\
e_{11}
\end{array}\right]^{T} R\left[\begin{array}{l}
e_{3} \\
e_{11}
\end{array}\right] \\
& \Sigma_{4}(h(k))=\Sigma_{41}-\Sigma_{42}-\Sigma_{43}(h(k)), \quad \Sigma_{41}=\left(e_{s}-e_{1}\right)^{T}\left(h_{1}^{2} Z_{1}+h_{12}^{2} Z_{2}\right)\left(e_{s}-e_{1}\right) \\
& \Sigma_{42}=E_{1}^{T}\left[\begin{array}{cc}
Z_{1} & 0 \\
* & 3 \rho\left(0, h_{1}\right) Z_{1}
\end{array}\right] E_{1}, \quad E_{1}=\left[\begin{array}{c}
e_{1}-e_{2} \\
e_{1}+e_{2}-2 e_{6}
\end{array}\right] \\
& \Sigma_{43}\left(h(k)=\left[\begin{array}{l}
E_{2} \\
E_{3}
\end{array}\right]^{T}\left(\left[\begin{array}{cc}
\tilde{Z}_{2} & S \\
* & \tilde{Z}_{2}
\end{array}\right]+\left[\begin{array}{cc}
\left(1-\delta_{h}\right) T_{1} & 0 \\
* & \delta_{h} T_{2}
\end{array}\right]\right)\left[\begin{array}{l}
E_{2} \\
E_{3}
\end{array}\right]\right. \\
& T_{1}=\tilde{Z}_{2}-S \tilde{Z}_{2}^{-1} S^{T}, \quad T_{2}=\tilde{Z}_{2}-S^{T} \tilde{Z}_{2}^{-1} S \\
& \tilde{\Sigma}_{43}\left(h_{1}\right)=\left[\begin{array}{c}
E_{2} \\
E_{3}
\end{array}\right]^{T}\left[\begin{array}{cc}
\tilde{Z}_{2} & S \\
* & \tilde{Z}_{2}
\end{array}\right]\left[\begin{array}{c}
E_{2} \\
E_{3}
\end{array}\right]+E_{2}^{T} \tilde{Z}_{2} E_{2} \\
& \tilde{\Sigma}_{43}\left(h_{2}\right)=\left[\begin{array}{c}
E_{2} \\
E_{3}
\end{array}\right]^{T}\left[\begin{array}{cc}
\tilde{Z}_{2} & S \\
* & \tilde{Z}_{2}
\end{array}\right]\left[\begin{array}{c}
E_{2} \\
E_{3}
\end{array}\right]+E_{3}^{T} \tilde{Z}_{2} E_{3} \\
& \tilde{Z}_{2}=\left[\begin{array}{cc}
Z_{2} & 0 \\
* & 3 Z_{2}
\end{array}\right], \quad E_{2}=\left[\begin{array}{c}
e_{2}-e_{3} \\
e_{2}+e_{3}-2 e_{7}
\end{array}\right] \text {, } \\
& E_{3}=\left[\begin{array}{c}
e_{3}-e_{4} \\
e_{3}+e_{4}-2 e_{8}
\end{array}\right], \quad E_{4}=\left[\begin{array}{c}
e_{2}-e_{5} \\
e_{2}+e_{5}-2 e_{9}
\end{array}\right] \\
& E_{5}(h(k))=\left[\begin{array}{c}
\left(h(k)-h_{1}+1\right) e_{7}-e_{2} \\
e_{2}-e_{3}
\end{array}\right], \quad E_{6}(h(k))=\left[\begin{array}{c}
\left(h_{2}-h(k)+1\right) e_{8}-e_{3} \\
e_{3}-e_{4}
\end{array}\right]
\end{aligned}
$$




$$
\begin{aligned}
& E_{56}(h(k))=\left[\begin{array}{l}
E_{5}(h(k)) \\
E_{6}(h(k))
\end{array}\right], \quad \Sigma_{51}(\alpha)=\left(e_{s}-e_{1}\right)^{T}\left(\alpha-h_{1}\right)^{2} Z_{3}\left(e_{s}-e_{1}\right) \\
& \Sigma_{52}(\alpha)=E_{4}^{T}\left[\begin{array}{cc}
Z_{3} & 0 \\
* & 3 \rho\left(h_{1}, \alpha\right) Z_{3}
\end{array}\right] E_{4}, \quad \Sigma_{6}=\Sigma_{61}-\Sigma_{62}(h(k)) \\
& K_{1}=\operatorname{diag}\left\{k_{1}^{-}, k_{2}^{-}, \cdots, k_{n}^{-}\right\}, \quad K_{2}=\operatorname{diag}\left\{k_{1}^{+}, k_{2}^{+}, \cdots, k_{n}^{+}\right\} \\
& \Sigma_{S}=\operatorname{Sym}\left\{\left(e_{10}-K_{1} e_{1}\right)^{T} M_{1}\left(e_{10}-K_{2} e_{1}\right)\right\}+\operatorname{Sym}\left\{\left(e_{11}-K_{1} e_{3}\right)^{T} M_{2}\left(e_{11}-K_{2} e_{3}\right)\right\} \\
& +\operatorname{Sym}\left\{\begin{array}{l}
\left(e_{10}-e_{11}-K_{1}\left(e_{1}-e_{3}\right)\right)^{T} M_{3} \times \\
\times\left(e_{10}-e_{11}-K_{2}\left(e_{1}-e_{3}\right)\right)
\end{array}\right\} \\
& \Sigma_{61}=h_{12}^{2}\left[\begin{array}{cc}
e_{1} \\
e_{s}-e_{1}
\end{array}\right]^{T}\left[\begin{array}{cc}
e_{1} \\
e_{s}-e_{1}
\end{array}\right]+h_{12} e_{2}^{T} H_{1} e_{2}-h_{12} e_{4}^{T} H_{2} e_{4}-h_{12} e_{3}^{T}\left(H_{1}-H_{2}\right) e_{3} \\
& \Sigma_{62}(h(k))=E_{56}^{T}(h(k)) G_{12} E_{56}(h(k)) \\
& G_{i}=G+\left[\begin{array}{ll}
0 & H_{i} \\
* & H_{i}
\end{array}\right], \quad i=1,2, \quad G_{12}=\left[\begin{array}{cc}
G_{1} & X \\
* & G_{2}
\end{array}\right]
\end{aligned}
$$

Theorem 1. [56] For given positive integers $h_{1}$ and $h_{2}$, system (1) with interval time-varying delay satisfying condition (2) is asymptotically stable, if there exist positive-definite matrices $P \in \mathfrak{R}^{4 n \times 4 n}, \quad Q_{i} \in \mathfrak{R}^{n \times n}, \quad i=1,2,3, \quad R \in \mathfrak{R}^{2 n \times 2 n} Z_{i} \in \mathfrak{R}^{n \times n}, \quad i=1,2,3$, positive definite diagonal matrices $M_{i} \in \mathfrak{R}^{n \times n}, i=1,2,3$ and any matrix $S \in \mathfrak{R}^{2 n \times 2 n}$ such that the following LMIs hold

$$
\begin{aligned}
& {\left[\begin{array}{cc}
\Phi\left(h_{1}, \alpha\right) & E_{2}^{T} S \\
* & -\tilde{Z}_{2}
\end{array}\right]<0} \\
& {\left[\begin{array}{cc}
\Phi\left(h_{2}, \alpha\right) & E_{3}^{T} S^{T} \\
* & -\tilde{Z}_{2}
\end{array}\right]<0}
\end{aligned}
$$

where

$\Phi\left(h_{i}, \alpha\right)=\Sigma_{11}+\Sigma_{12}\left(h_{i}, \alpha\right)+\Sigma_{2}+\Sigma_{3}+\Sigma_{41}-\Sigma_{42}-\tilde{\Sigma}_{43}\left(h_{i}\right)+\Sigma_{51}(\alpha)-\Sigma_{52}(\alpha)-\Sigma_{s}, \quad i=1,2$

Proof. Construct a LKF for the DNN (1) as follows:

$$
V(k)=\sum_{i=1}^{5} V_{i}(k)
$$

where

$$
\begin{aligned}
& V_{1}(k)=\zeta_{1}^{T}(k) P \zeta_{1}(k) \\
& V_{2}(k)=\sum_{j=k-h_{1}}^{k-1} x^{T}(j) Q_{1} x(j)+\sum_{j=k-h_{2}}^{k-\alpha-1} x^{T}(j) Q_{2} x(j)+\sum_{j=k-\alpha}^{k-h_{1}-1} x^{T}(j) Q_{3} x(j) \\
& V_{3}(k)=\sum_{j=k-h(k)}^{k-1} \zeta_{2}^{T}(j) R \zeta_{2}(j)+\sum_{i=-h_{2}+1}^{-h_{1}} \sum_{j=k+i}^{k-1} \zeta_{2}^{T}(j) R \zeta_{2}(j)
\end{aligned}
$$




$$
\begin{aligned}
& V_{4}(k)=h_{1} \sum_{i=-h_{1}}^{-1} \sum_{j=k+i}^{k-1} \eta^{T}(j) Z_{1} \eta(j)+h_{12} \sum_{i=-h_{2}}^{-h_{1}-1} \sum_{j=k+i}^{k-1} \eta^{T}(j) Z_{2} \eta(j) \\
& V_{5}(k)=\left(\alpha-h_{1}\right) \sum_{i=-\alpha}^{-h_{1}-1} \sum_{j=k+i}^{k-1} \eta^{T}(j) Z_{3} \eta(j)
\end{aligned}
$$

The forward difference of $\operatorname{LKF}(31), \Delta V(k)=V(k+1)-V(k)$, along the trajectories of the DNN (1) gives

$$
\Delta V_{1}(k)=\zeta_{1}^{T}(k+1) P \zeta_{1}(k+1)-\zeta_{1}^{T}(k) P \zeta_{1}(k)
$$

Since

$$
\begin{gathered}
\zeta_{1}(k)=\operatorname{Col}\left\{\begin{array}{l}
e_{1},\left(h_{1}+1\right) e_{6}-e_{1}, \\
\left(h(k)-h_{1}+1\right) e_{7}+\left(h_{2}-h(k)+1\right) e_{8} \\
-e_{2}-e_{3}, \quad\left(\alpha-h_{1}+1\right) e_{9}-e_{2}
\end{array}\right\} \xi(k)=\left(\Pi_{2}+\Pi(h(k), \alpha)\right) \xi(k) \\
\zeta_{1}(k+1)=\operatorname{Col}\left\{\begin{array}{l}
e_{s},\left(h_{1}+1\right) e_{6}-e_{2}, \\
\left(h(k)-h_{1}+1\right) e_{7} \\
+\left(h_{2}-h(k)+1\right) e_{8}-e_{3}-e_{4} \\
\left(\alpha-h_{1}+1\right) e_{9}-e_{5}
\end{array}\right\} \xi(k)=\left(\Pi_{1}+\Pi(h(k), \alpha)\right) \xi(k)
\end{gathered}
$$

it follows

$$
\Delta V_{1}(k)=\xi^{T}(k)\left(\Sigma_{11}+\Sigma_{12}(h(k), \alpha)\right) \xi(k)
$$

The forward differences of $V_{2}(k)$ and $V_{3}(k)$ can be obtained as

$$
\begin{aligned}
\Delta V_{2}(k) & =\xi^{T}(k)\left\{e_{1}^{T} Q_{1} e_{1}+e_{2}^{T}\left(Q_{3}-Q_{1}\right) e_{2}+e_{5}^{T}\left(Q_{2}-Q_{3}\right) e_{5}-e_{4}^{T} Q_{2} e_{4}\right\} \xi(k) \\
& =\xi^{T}(k) \Sigma_{2} \xi(k) \\
\Delta V_{3}(k) & =\sum_{j=k+1-h(k+1)}^{k-h_{1}} \zeta_{2}^{T}(j) R \zeta_{2}(j)+\sum_{j=k-h_{1}+1}^{k-1} \zeta_{2}^{T}(j) R \zeta_{2}(j) \\
& +\zeta_{2}^{T}(k) R \zeta_{2}(k)-\sum_{j=k-h(k)}^{k-1} \zeta_{2}^{T}(j) R \zeta_{2}(j) \\
& +\sum_{i=-h_{2}+1}^{-h_{1}}\left[\sum_{j=k+i}^{k-1} \zeta_{2}^{T}(j) R \zeta_{2}(j)+\zeta_{2}^{T-1}(k) R \zeta_{2}(k)-\zeta_{2}^{T}(k+i) R \zeta_{2}(k+i)\right) \\
& \leq \xi^{T}(k)\left[\begin{array}{c}
e_{1} \\
e_{10}
\end{array}\right]^{T}\left(h_{12}+1\right) R\left[\begin{array}{c}
e_{1} \\
e_{10}
\end{array}\right] \xi(k)-\xi^{T}(k)\left[\begin{array}{c}
e_{3} \\
e_{11}
\end{array}\right]^{T} R\left[\begin{array}{c}
e_{3} \\
e_{11}
\end{array}\right] \xi(k) \\
& =\xi^{T}(k) \Sigma_{3} \xi(k)
\end{aligned}
$$




$$
\begin{aligned}
\Delta V_{4}(k)= & h_{1} \sum_{i=-h_{1}}^{-1}\left(\eta^{T}(k) Z_{1} \eta(k)-\eta^{T}(k+i) Z_{1} \eta(k+i)\right) \\
& +h_{12} \sum_{i=-h_{2}}^{-h_{1}-1}\left(\eta^{T}(k) Z_{2} \eta(k)-\eta^{T}(k+i) Z_{2} \eta(k+i)\right) \\
= & \xi^{T}(k)\left(e_{s}-e_{1}\right)^{T}\left(h_{1}^{2} Z_{1}+h_{12}^{2} Z_{2}\right)\left(e_{s}-e_{1}\right) \xi(k) \\
& -h_{1} \sum_{j=k-h_{1}}^{k-1} \eta^{T}(j) Z_{1} \eta(j)-h_{12} \sum_{j=k-h_{2}}^{k-h_{1}-1} \eta^{T}(j) Z_{2} \eta(j)
\end{aligned}
$$

Using inequality (7) from Lemma 2 to estimate $Z_{1}$-dependent summation term yields

$$
h_{1} \sum_{j=k-h_{1}}^{k-1} \eta^{T}(j) Z_{1} \eta(j) \geq\left[\begin{array}{l}
\omega_{1} \\
\omega_{2}
\end{array}\right]^{T}\left[\begin{array}{cc}
Z_{1} & 0 \\
* & 3 \rho\left(0, h_{1}\right) Z_{1}
\end{array}\right]\left[\begin{array}{l}
\omega_{1} \\
\omega_{2}
\end{array}\right]=\xi^{T}(k) \Sigma_{42} \xi(k)
$$

Similarly, by using (7) we estimate $Z_{2}$-dependent summation term

$$
\begin{gathered}
h_{12} \sum_{j=k-h_{2}}^{k-h_{1}-1} \eta^{T}(j) Z_{2} \eta(j)=h_{12} \sum_{j=k-h_{2}}^{k-h(k)-1} \eta^{T}(j) Z_{2} \eta(j)+h_{12} \sum_{j=k-h(k)}^{k-h_{1}-1} \eta^{T}(j) Z_{2} \eta(j) \\
\geq \frac{1}{\delta_{h}} \xi^{T}(k) E_{2}^{T}\left[\begin{array}{cc}
Z_{2} & 0 \\
0 & 3 Z_{2}
\end{array}\right] E_{2} \xi(k)+\frac{1}{1-\delta_{h}} \xi^{T}(k) E_{3}^{T}\left[\begin{array}{rr}
Z_{2} & 0 \\
0 & 3 Z_{2}
\end{array}\right] E_{3} \xi(k) \\
=\xi^{T}(k)\left[\begin{array}{l}
E_{2} \\
E_{3}
\end{array}\right]^{T}\left[\begin{array}{cc}
\frac{1}{\delta_{h}} \tilde{Z}_{2} & 0 \\
0 & \frac{1}{1-\delta_{h}} \tilde{Z}_{2}
\end{array}\right]\left[\begin{array}{l}
E_{2} \\
E_{3}
\end{array}\right] \xi(k)
\end{gathered}
$$

Based on Lemma 4, for any matrix $S \in \mathfrak{R}^{2 n \times 2 n}$ we have

$$
\begin{aligned}
h_{12} \sum_{j=k-h_{2}}^{k-h_{1}-1} \eta^{T}(j) Z_{2} \eta(j) & \geq \xi^{T}(k)\left[\begin{array}{c}
E_{2} \\
E_{3}
\end{array}\right]^{T}\left[\begin{array}{cc}
\tilde{Z}_{2}+\left(1-\delta_{h}\right) T_{1} & S \\
* & \tilde{Z}_{2}+\delta_{h} T_{2}
\end{array}\right]\left[\begin{array}{c}
E_{2} \\
E_{3}
\end{array}\right] \xi(k) \\
& =\xi^{T}(k) \Sigma_{43}\left(h(k) \xi^{T}(k)\right.
\end{aligned}
$$

Then, we can get the upper bound of $\Delta V_{4}(k)$ as

$$
\Delta V_{4}(k) \leq \xi^{T}(k)\left\{\Sigma_{41}-\Sigma_{42}-\Sigma_{43}(h(k))\right\} \xi(k)=\xi^{T}(k) \Sigma_{4}(h(k)) \xi(k)
$$

Calculating $\Delta V_{5}(k)$ gives

$$
\begin{aligned}
\Delta V_{5}(k) & =\left(\alpha-h_{1}\right)^{2} \eta^{T}(k) Z_{3} \eta(k)-\left(\alpha-h_{1}\right) \sum_{i=-\alpha}^{-h_{1}-1} \eta^{T}(k+i) Z_{3} \eta(k+i) \\
& =\xi^{T}(k)\left(e_{s}-e_{1}\right)^{T}\left(\alpha-h_{1}\right)^{2} Z_{3}\left(e_{s}-e_{1}\right) \xi(k)-\left(\alpha-h_{1}\right) \sum_{j=k-\alpha}^{k-h_{1}-1} \eta^{T}(j) Z_{3} \eta(j)
\end{aligned}
$$

By inequality (7), the second term of $\Delta V_{5}(k)$ can be written as

$$
\left(\alpha-h_{1}\right) \sum_{j=k-\alpha}^{k-h_{1}-1} \eta^{T}(j) Z_{3} \eta(j) \geq\left[\begin{array}{l}
\omega_{3} \\
\omega_{4}
\end{array}\right]^{T}\left[\begin{array}{cc}
Z_{3} & 0 \\
* & 3 \rho\left(h_{1}, \alpha\right) Z_{3}
\end{array}\right]\left[\begin{array}{l}
\omega_{3} \\
\omega_{4}
\end{array}\right]
$$


Then, we can get the upper bound of $\Delta V_{5}(k)$ as

$$
\Delta V_{5}(k) \leq \xi^{T}(k)\left(\begin{array}{l}
\Sigma_{51}(\alpha) \\
-E_{4}^{T}\left[\begin{array}{cc}
Z_{3} & 0 \\
* & 3 \rho\left(h_{1}, \alpha\right) Z_{3}
\end{array}\right] E_{4}
\end{array}\right) \xi(k)=\xi^{T}(k)\left(\Sigma_{51}(\alpha)-\Sigma_{52}(\alpha)\right) \xi(k)
$$

Under the assumption on the activation function (4) and (5), for any positive definite diagonal matrices $M_{j}=\operatorname{diag}\left\{m_{j 1}, m_{j 2}, \cdots, m_{j n}\right\}, j=1,2,3$, the following inequality holds

$$
\begin{aligned}
& 0 \geq 2 \sum_{i=1}^{n} m_{1 i}\left[f_{i}\left(x_{i}(k)\right)-k_{i}^{-} x_{i}(k)\right] \times\left[f_{i}\left(x_{i}(k)\right)-k_{i}^{+} x_{i}(k)\right] \\
&+2 \sum_{i=1}^{n} m_{2 i}\left[f_{i}\left(x_{i}(k-h(k))\right)-k_{i}^{-} x_{i}(k-h(k))\right]\left[f_{i}\left(x_{i}(k-h(k))\right)-k_{i}^{+} x_{i}(k-h(k))\right] \\
&+2 \sum_{i=1}^{n} m_{3 i}\left[\begin{array}{l}
f_{i}\left(x_{i}(k)\right)-f_{i}\left(x_{i}(k-h(k))\right) \\
-k_{i}^{-}\left(x_{i}(k)-x_{i}(k-h(k))\right)
\end{array}\right]\left[\begin{array}{l}
f_{i}\left(x_{i}(k)\right)-f_{i}\left(x_{i}(k-h(k))\right) \\
-k_{i}^{+}\left(x_{i}(k)-x_{i}(k-h(k))\right)
\end{array}\right]
\end{aligned}
$$

Then

$$
\begin{aligned}
& 0 \geq 2 {\left[f(x(k))-K_{1} x(k)\right] M_{1}\left[f(x(k))-K_{2} x(k)\right] } \\
&+ 2\left[f(x(k-h(k)))-K_{1} x(k-h(k))\right] M_{2}\left[f(x(k-h(k)))-K_{2} x(k-h(k))\right] \\
&+2\left[\begin{array}{l}
f(x(k))-f(x(k-h(k))) \\
-K_{1}(x(k)-x(k-h(k)))
\end{array}\right] M_{3}\left[\begin{array}{l}
f(x(k))-f(x(k-h(k)))] \\
-K_{2}(x(k)-x(k-h(k)))
\end{array}\right] \\
& 0 \geq 2 \xi^{T}(k)\left(e_{10}-K_{1} e_{1}\right)^{T} M_{1}\left(e_{10}-K_{2} e_{1}\right) \xi^{T}(k) \\
&+2 \xi^{T}(k)\left(e_{11}-K_{1} e_{3}\right)^{T} M_{2}\left(e_{11}-K_{2} e_{3}\right) \xi^{T}(k) \\
&+2 \xi^{T}(k)\left(e_{10}-e_{11}-K_{1}\left(e_{1}-e_{3}\right)\right)^{T} M_{3}\left(e_{10}-e_{11}-K_{2}\left(e_{1}-e_{3}\right)\right) \xi^{T}(k)
\end{aligned}
$$

So, we have

$$
\xi^{T}(k) \Sigma_{S} \xi(k) \leq 0
$$

Therefore, by combining (35)-(37), (42), (45) and (49), the forward difference of $V(k)$ is obtained as

$$
\Delta V(k) \leq \xi^{T}(k) \Sigma(h(k), \alpha) \xi(k)
$$

Since the matrix $\Sigma(h(k), \alpha)$ is affine with respect to the delay $h(k)$, the condition $\Sigma(h(k), \alpha)<0$ is satisfied if and only if

$$
\Sigma\left(h_{i}, \alpha\right)<0, \quad i=1,2
$$

By calculating, we have

$$
\begin{aligned}
& \Sigma_{12}\left(h_{1}, \alpha\right)=\operatorname{Sym}\left\{\left(\Pi_{1}-\Pi_{2}\right)^{T} P \Pi\left(h_{1}, \alpha\right)\right\} \\
& \Sigma_{12}\left(h_{2}, \alpha\right)=\operatorname{Sym}\left\{\left(\Pi_{1}-\Pi_{2}\right)^{T} P \Pi\left(h_{2}, \alpha\right)\right\}
\end{aligned}
$$




$$
\begin{aligned}
\Sigma_{43}\left(h_{1}\right) & =\left[\begin{array}{l}
E_{2} \\
E_{3}
\end{array}\right]^{T}\left(\left[\begin{array}{cc}
\tilde{Z}_{2} & S \\
* & \tilde{Z}_{2}
\end{array}\right]+\left[\begin{array}{cc}
T_{1} & 0 \\
* & 0
\end{array}\right]\right)\left[\begin{array}{l}
E_{2} \\
E_{3}
\end{array}\right] \\
& =\left[\begin{array}{c}
E_{2} \\
E_{3}
\end{array}\right]^{T}\left[\begin{array}{cc}
\tilde{Z}_{2} & S \\
* & \tilde{Z}_{2}
\end{array}\right]\left[\begin{array}{c}
E_{2} \\
E_{3}
\end{array}\right]+E_{2}^{T} \tilde{Z}_{2} E_{2}-E_{2}^{T} S \tilde{Z}_{2}^{-1} S^{T} E_{2} \\
& =\tilde{\Sigma}_{43}\left(h_{1}\right)-E_{2}^{T} S \tilde{Z}_{2}^{-1} S^{T} E_{2} \\
\Sigma_{43}\left(h_{2}\right) & =\left[\begin{array}{c}
E_{2} \\
E_{3}
\end{array}\right]^{T}\left(\left[\begin{array}{cc}
\tilde{Z}_{2} & S \\
* & \tilde{Z}_{2}
\end{array}\right]+\left[\begin{array}{cc}
0 & 0 \\
* & T_{2}
\end{array}\right]\right)\left[\begin{array}{l}
E_{2} \\
E_{3}
\end{array}\right] \\
& =\left[\begin{array}{l}
E_{2} \\
E_{3}
\end{array}\right]^{T}\left[\begin{array}{cc}
\tilde{Z}_{2} & S \\
* & \tilde{Z}_{2}
\end{array}\right]\left[\begin{array}{l}
E_{2} \\
E_{3}
\end{array}\right]+E_{3}^{T} \tilde{Z}_{2} E_{3}-E_{3}^{T} S^{T} \tilde{Z}_{2}^{-1} S E_{3} \\
& =\tilde{\Sigma}_{43}\left(h_{2}\right)-E_{3}^{T} S^{T} \tilde{Z}_{2}^{-1} S E_{3} \\
\Sigma\left(h_{1}, \alpha\right) & =\Phi\left(h_{1}, \alpha\right)+E_{2}^{T} S \tilde{Z}_{2}^{-1} S^{T} E_{2}<0 \\
\Sigma\left(h_{2}, \alpha\right) & =\Phi\left(h_{2}, \alpha\right)+E_{3}^{T} S^{T} \tilde{Z}_{2}^{-1} S E_{3}<0
\end{aligned}
$$

Then, by taking Schur complement, it can be seen that (55) and (56) are equivalent to (29) and (30), respectively. Therefore, when (29) and (30) hold, $\Delta V(k)<0$, which shows that system (1) is asymptotically stable.

Remark 4. In Theorem 1, the Wirtinger-based summation inequality (7) is applied to summation with the constant lower and upper bound $\left(h_{1} \sum_{j=k-h_{1}}^{k-1} \eta^{T}(j) Z_{1} \eta(j)\right)$. However, in the case of the summation with the time-varying lower or upper bound

$$
h_{12} \sum_{j=k-h_{2}}^{k-h(k)-1} \eta^{T}(j) Z_{2} \eta(j)+h_{12} \sum_{j=k-h(k)}^{k-h_{1}-1} \eta^{T}(j) Z_{2} \eta(j)
$$

combination of the Wirtinger-based inequality (7) and the reciprocally convex approach (11) is applied.

By introducing an augmented LKF and zero equations, a further improved stability condition of system (1) can be obtained as follows.

Theorem 2. [56] For given positive integers $h_{1}$ and $h_{2}$, system (1) with interval time-varying delay satisfying condition (2) is asymptotically stable, if there exist positive-definite matrices $P \in \mathfrak{R}^{4 n \times 4 n}, Q_{i} \in \mathfrak{R}^{n \times n}, i=1,2,3, R \in \mathfrak{R}^{2 n \times 2 n} Z_{i} \in \mathfrak{R}^{n \times n}, i=1,2,3, G \in \mathfrak{R}^{2 n \times 2 n}$, positive definite diagonal matrices $M_{i} \in \mathfrak{R}^{n \times n}, i=1,2,3$, symmetric matrices $H_{i} \in \mathfrak{R}^{n \times n}, i=1,2$ and any matrices $S \in \mathfrak{R}^{2 n \times 2 n}$ and $X \in \mathfrak{R}^{2 n \times 2 n}$ such that the following LMIs hold

$$
\begin{aligned}
& {\left[\begin{array}{cc}
G_{1} & X \\
* & G_{2}
\end{array}\right]>0} \\
& {\left[\begin{array}{cc}
\Phi\left(h_{1}, \alpha\right)+\Sigma_{61}-\Sigma_{62}\left(h_{1}\right) & E_{2}^{T} S \\
* & -\tilde{Z}_{2}
\end{array}\right]<0}
\end{aligned}
$$




$$
\left[\begin{array}{cc}
\Phi\left(h_{2}, \alpha\right)+\Sigma_{61}-\Sigma_{62}\left(h_{2}\right) & E_{3}^{T} S^{T} \\
* & -\tilde{Z}_{2}
\end{array}\right]<0
$$

where

$\Phi\left(h_{i}, \alpha\right)=\Sigma_{11}+\Sigma_{12}\left(h_{i}, \alpha\right)+\Sigma_{2}+\Sigma_{3}+\Sigma_{41}-\Sigma_{42}-\tilde{\Sigma}_{43}\left(h_{i}\right)+\Sigma_{51}(\alpha)-\Sigma_{52}(\alpha)-\Sigma_{s}, \quad i=1,2$.

Proof. Construct the following LKF candidate

$$
\tilde{V}(k)=V(k)+V_{6}(k)
$$

where

$$
V_{6}(k)=h_{12} \sum_{i=-h_{2}}^{-h_{1}-1} \sum_{j=k+i}^{k-1} \zeta_{3}^{T}(j) G \xi_{3}(j)
$$

The forward difference of (62) along the trajectories of DNN (1) gives

$$
\Delta V_{6}(k)=h_{12}^{2} \zeta_{3}^{T}(k) G \xi_{3}(k)-h_{12} \sum_{j=k-h_{2}}^{k-h(k)-1} \zeta_{3}^{T}(j) G \xi_{3}(j)-h_{12} \sum_{j=k-h(k)}^{k-h_{1}-1} \zeta_{3}^{T}(j) G \xi_{3}(j)
$$

For any symmetric matrices $H_{i}, i=1,2$ we have

$$
\begin{aligned}
& \sum_{j=k-l_{2}}^{k-l_{1}-1}\left(x^{T}(j+1) H_{i} x(j+1)-x^{T}(j) H_{i} x(j)\right) \\
& \quad=x^{T}\left(k-l_{1}\right) H_{i} x\left(k-l_{1}\right)-x^{T}\left(k-l_{2}\right) H_{i} x\left(k-l_{2}\right)
\end{aligned}
$$

Since

$$
x^{T}(j+1) H_{i} x(j+1)-x^{T}(j) H_{i} x(j)=\left[\begin{array}{c}
x(j) \\
\eta(j)
\end{array}\right]^{T}\left[\begin{array}{cc}
0 & H_{i} \\
* & H_{i}
\end{array}\right]\left[\begin{array}{l}
x(j) \\
\eta(j)
\end{array}\right]=\zeta_{3}^{T}(k)\left[\begin{array}{cc}
0 & H_{i} \\
* & H_{i}
\end{array}\right] \xi_{3}(j)
$$

then the following zero equation holds

$$
\begin{aligned}
E Q\left(l_{1}, l_{2}, H_{i}\right) & =x^{T}\left(k-l_{1}\right) H_{i} x\left(k-l_{1}\right)-x^{T}\left(k-l_{2}\right) H_{i} x\left(k-l_{2}\right) \\
& -\sum_{j=k-l_{2}}^{k-l_{1}-1} \zeta_{3}^{T}(k)\left[\begin{array}{cc}
0 & H_{i} \\
* & H_{i}
\end{array}\right] \xi_{3}(j)=0
\end{aligned}
$$

Now, from (66) we get the following two zero equations

$$
\begin{aligned}
E Q\left(h_{1}, h(k), H_{1}\right)= & x^{T}\left(k-h_{1}\right) H_{1} x\left(k-h_{1}\right)-x^{T}(k-h(k)) H_{1} x(k-h(k)) \\
& -\sum_{j=k-h(k)}^{k-h_{1}-1} \zeta_{3}^{T}(k)\left[\begin{array}{cc}
0 & H_{1} \\
* & H_{1}
\end{array}\right] \xi_{3}(j)=0 \\
E Q\left(h(k), h_{2}, H_{2}\right)= & x^{T}(k-h(k)) H_{2} x(k-h(k))-x^{T}\left(k-h_{2}\right) H_{2} x\left(k-h_{2}\right) \\
& -\sum_{j=k-h_{2}}^{k-h(k)-1} \zeta_{3}^{T}(k)\left[\begin{array}{ll}
0 & H_{2} \\
* & H_{2}
\end{array}\right] \xi_{3}(j)=0
\end{aligned}
$$


Adding the inequality (67) and (68) to (63) implies

$$
\begin{aligned}
\Delta V_{6}(k)= & h_{12}^{2} \zeta_{3}^{T}(k) G \xi_{3}(k)+h_{12} x^{T}\left(k-h_{1}\right) H_{1} x\left(k-h_{1}\right) \\
& -h_{12} x^{T}(k-h(k))\left(H_{1}-H_{2}\right) x(k-h(k))-h_{12} x^{T}\left(k-h_{2}\right) H_{2} x\left(k-h_{2}\right) \\
& -h_{12} \sum_{j=k-h(k)}^{k-h_{1}-1} \zeta_{3}^{T}(j) G_{1} \xi_{3}(j)-h_{12} \sum_{j=k-h_{2}}^{k-h(k)-1} \zeta_{3}^{T}(j) G_{2} \xi_{3}(j)
\end{aligned}
$$

Applying Jensen inequality (6), the $\Delta V_{6}(k)$ is estimated as

$$
\begin{gathered}
\Delta V_{6}(k) \leq \xi^{T}(k)\left\{\begin{array}{c}
h_{12}^{2}\left[\begin{array}{c}
e_{1} \\
e_{s}-e_{1}
\end{array}\right]^{T} G\left[\begin{array}{c}
e_{1} \\
e_{s}-e_{1}
\end{array}\right] \\
+h_{12} e_{2}^{T} H_{1} e_{2}-h_{12} e_{4}^{T} H_{2} e_{4}-h_{12} e_{3}^{T}\left(H_{1}-H_{2}\right) e_{3}
\end{array}\right\} \xi(k) \\
-\frac{1}{\delta_{h}} \sum_{j=k-h(k)}^{k-h_{1}-1} \zeta_{3}^{T}(j) G_{1} \sum_{j=k-h(k)}^{k-h_{1}-1} \xi_{3}(j)-\frac{1}{1-\delta_{h}} \sum_{j=k-h_{2}}^{k-h(k)-1} \zeta_{3}^{T}(j) G_{2} \sum_{j=k-h_{2}}^{k-h(k)-1} \xi_{3}(j) \\
=\xi^{T}(k) \Sigma_{61} \xi(k)-\frac{1}{\delta_{h}} \xi^{T}(k) E_{5}^{T}(h(k)) G_{1} E_{5}(h(k)) \xi(k) \\
-\frac{1}{1-\delta_{h}} \xi^{T}(k) E_{6}^{T}(h(k)) G_{2} E_{6}(h(k)) \xi(k)
\end{gathered}
$$

Using Lemma 3, for any matrix $X \in \mathfrak{R}^{2 n \times 2 n}$ we have

Finally

$$
\begin{aligned}
\Delta V_{6} & (k) \leq \xi^{T}(k)\left(\Sigma_{61}-E_{56}^{T}(h(k))\left[\begin{array}{cc}
G_{1} & X \\
* & G_{2}
\end{array}\right] E_{56}(h(k))\right) \xi(k) \\
= & \xi^{T}(k)\left(\Sigma_{61}-E_{56}^{T}(h(k)) G_{12} E_{56}(h(k))\right) \xi(k) \\
= & \xi^{T}(k)\left[\Sigma_{61}-\Sigma_{62}(h(k))\right] \xi(k)=\xi^{T}(k) \Sigma_{6}(h(k)) \xi(k)
\end{aligned}
$$

$$
\left.\Delta \tilde{V}(k) \leq \xi^{T}(k)[\Sigma(h(k)), \alpha)+\Sigma_{61}-\Sigma_{62}(h(k))\right] \xi(k)
$$

Based on Lemma 5, the equivalent condition of $\Sigma(h(k)), \alpha)+\Sigma_{61}-E_{56}^{T}(h(k)) G_{12} E_{56}(h(k))<0$ is that exists a matrix $\Psi$ such that

$$
\Theta(h(k), \alpha)=\left[\begin{array}{c:c}
\Sigma(h(k)), \alpha)+\Sigma_{61} & \Psi^{T} \\
+\operatorname{Sym}\left\{E_{56}^{T}(h(k)) \Psi\right\} & \\
\hdashline * & -G_{12}
\end{array}\right]<0
$$

Since the matrix $\Theta(h(k), \alpha)$ is affine with respect to the delay $h(k)$, the condition $\Theta(h(k), \alpha)<0$ is satisfied if and only if

$$
\Theta\left(h_{i}, \alpha\right)<0, \quad i=1,2
$$

Based on Lemma 5, (74) is equivalent to i.e.

$$
\Sigma\left(h_{i}, \alpha\right)+\Sigma_{61}-E_{56}^{T}\left(h_{i}\right) G_{12} E_{56}\left(h_{i}\right)=\Sigma\left(h_{i}, \alpha\right)+\Sigma_{61}-\Sigma_{62}\left(h_{i}\right)<0, \quad i=1,2
$$

$$
\begin{aligned}
& \Phi\left(h_{1}, \alpha\right)+E_{2}^{T} S \tilde{Z}_{2}^{-1} S^{T} E_{2}+\Sigma_{61}-\Sigma_{62}\left(h_{1}\right)<0 \\
& \Phi\left(h_{2}, \alpha\right)+E_{3}^{T} S^{T} \tilde{Z}_{2}^{-1} S E_{3}+\Sigma_{61}-\Sigma_{62}\left(h_{2}\right)<0
\end{aligned}
$$


Then, by taking the Schur complement, it can be seen that (76) and (77) are equivalent to (59) and (60), respectively. Therefore, when (59) and (60) hold, $\Delta \tilde{V}(k)<0$, which shows that system (1) is asymptotically stable.

Remark 5. In order to improve the stability criterion proposed in Theorem 1 , in the proof of Theorem 2 a new double summation term $V_{6}(k)$ (with free matrix $G$ ) is introduced in LKF and two zero equalities (with symmetric matrices $H_{1}$ and $H_{2}$ ) are introduced in $\Delta V_{6}(k)$. In this way, the conservatism of the Theorem 2 is reduced.

Remark 6. In order to reduce the number of decision variables, Lemma 5 is used twice. First, by using the lemma, non-affine term

$$
\Sigma(h(k)), \alpha)+\Sigma_{61}-E_{56}^{T}(h(k)) G_{12} E_{56}(h(k))<0
$$

with respect to $h(k)$ is transformed into the affine term $\Theta(h(k), \alpha)$. On that occasion, additional matrix $\Psi \in \mathfrak{R}^{11 n \times 2 n}$ is introduced in (73) and the number of decision variables have increased significantly (for $22 n^{2}$ ). Second, if we apply Lemma 5 on (74), then the matrix $\Psi \in \mathfrak{R}^{11 n \times 2 n}$ can be eliminated from $\Theta\left(h_{i}, \alpha\right)$. In this way, the significantly reduction of decision variables was performed.

Remark 7. The proposed stability criteria depend on the lower and upper bounds of timedelay, $h_{1}$ and $h_{2}$. In order to compare proposed results with existing ones, we calculate a maximum allowable upper bound (MAUB) of the time-delay, $h_{\max }=h_{2 \max }$ such that the concerned system is asymptotically stable for any delay size less than the MAUB. Note that a criterion that gives a lower value of MAUB is less conservative with respect to other criteria.

\subsection{FTS and FTP for DNNs with time-varying delay}

In this section, we present FTS and FTP for DNN (1) with time-varying delay using the weighted summation inequalities (17)-(19) [16].

Theorem 3. [16] Under Assumption 1, DNN (1) is finite time stable with respect to $(\alpha, \beta, N)$, where $0 \leq \alpha<\beta$, if there exist a scalar $\gamma>1$, positive scalars $\lambda_{i}, i=1,2, \ldots, 9$, positive definite matrices $P, Q_{1}, Q_{2}, Q_{3}, R, Z_{1}, Z_{2}$, and $Z_{3}$, positive definite diagonal matrices $\Lambda_{1}$ and $\Lambda_{2}$, matrices $U, V$ and $\Omega=[\Omega 1 \Omega 2]$, such that the following LMIs hold:

$$
\begin{aligned}
& {\left[\begin{array}{ccc}
\Sigma+\Gamma_{i} \Omega^{T}+\Omega \Gamma_{i}^{T} & \Omega_{1} & \Omega_{2} \\
* & -Z_{2} & -U \\
* & * & -Z_{2}
\end{array}\right]<0, \quad i=1,2} \\
& {\left[\begin{array}{cc}
Z_{1} & V \\
* & Z_{1}
\end{array}\right]>0, \quad\left[\begin{array}{cc}
Z_{2} & U \\
* & Z_{2}
\end{array}\right]>0,} \\
& \lambda_{1} I<P<\lambda_{2} I, \quad Q_{1}<\lambda_{3} I, \quad Q_{2}<\lambda_{4} I, \quad Q_{3}<\lambda_{5} I, \\
& R<\lambda_{6} I, \quad Z_{1}<\lambda_{7} I, \quad Z_{2}<\lambda_{8} I, \quad Z_{3}<\lambda_{9} I \\
& \gamma^{N}\left[\begin{array}{l}
\alpha\left(\lambda_{2}+\varepsilon_{1} \lambda_{3}+\varepsilon_{2} \lambda_{4}+\left(\varepsilon_{2}+\varepsilon_{3}\right) \lambda_{5}\right)+\left(\varepsilon_{2}+\varepsilon_{3}\right) \hat{f}^{2} \lambda_{6} \\
+\delta\left(\pi \varepsilon_{4} \lambda_{7}+\kappa \varepsilon_{5} \lambda_{8}+\vartheta \varepsilon_{6} \lambda_{9}\right)
\end{array}\right]-\beta \lambda_{1}<0,
\end{aligned}
$$


where

$$
\begin{aligned}
& \Sigma \in \mathfrak{R}^{8 n \times 8 n} \\
& \Sigma_{11}=C^{T} P C-\gamma P+Q_{1}+Q_{2}+\left(h_{2}-h_{1}+1\right) Q_{3}+(C-I)^{T} Z_{123}(C-I)-F_{1} \Lambda_{1}, \\
& \Sigma_{15}=C^{T} P A+(C-I)^{T} Z_{123} A+F_{2} \Lambda_{1} \text {, } \\
& \Sigma_{16}=C^{T} P A_{d}+(C-I)^{T} Z_{123} A_{d}, \quad \Sigma_{22}=-\gamma^{h_{1}} Q_{1}-Z_{1}-h_{21}^{2} Z_{3}, \quad \Sigma_{23}=Z_{1}-V^{T}, \\
& \Sigma_{24}=V^{T}, \quad \Sigma_{27}=h_{21} Z_{3}, \quad \Sigma_{28}=h_{21} Z_{3}, \quad \Sigma_{33}=-\gamma^{h_{1}} Q_{3}-2 Z_{1}+V+V^{T}-F_{1} \Lambda_{2} \text {, } \\
& \Sigma_{34}=Z_{1}-V^{T}, \quad \Sigma_{36}=F_{2} \Lambda_{2}, \quad \Sigma_{44}=-\gamma^{h_{2}} Q_{2}-Z_{1}, \\
& \Sigma_{55}=A^{T} P A+\left(h_{2}-h_{1}+1\right) R+A^{T} Z_{123} A-\Lambda_{1} \text {, } \\
& \Sigma_{56}=A^{T} P A_{d}+A^{T} Z_{123} A_{d}, \quad \Sigma_{66}=-\gamma^{h_{1}} R+A_{d}^{T} P A_{d}+A_{d}^{T} Z_{123} A_{d}-\Lambda_{2}, \\
& \Sigma_{77}=-Z_{3}, \quad \Sigma_{78}=-Z_{3}, \quad \Sigma_{88}=-Z_{3} \\
& \Gamma_{1}=\left[\begin{array}{cccc}
h_{21} I_{n} & 0_{n \times 5 n} & -I_{n} & 0_{n} \\
0_{n} & 0_{n \times 5 n} & 0_{n} & -I_{n}
\end{array}\right]^{T}, \quad \Gamma_{2}=\left[\begin{array}{cccc}
0_{n} & 0_{n \times 5 n} & -I_{n} & 0_{n} \\
h_{21} I_{n} & 0_{n \times 5 n} & 0_{n} & -I_{n}
\end{array}\right]^{T}, \\
& Z_{12}=\pi h_{21} Z_{1}+\kappa \tau_{1} Z_{2}, \quad Z_{123}=Z_{12}+\vartheta \tau_{2} Z_{3} \text {, } \\
& \hat{f}=\max _{1 \leq i \leq n}\left\{\left|k_{i}^{-}\right|,\left|k_{i}^{+}\right|\right\} \\
& \Lambda_{i}=\operatorname{diag}\left\{\lambda_{i 1}, \lambda_{i 2}, \cdots, \lambda_{i n}\right\}, \quad i=1,2 \\
& F_{1}=\operatorname{diag}\left\{k_{1}^{-} k_{1}^{+}, k_{2}^{-} k_{2}^{+}, \cdots, k_{n}^{-} k_{n}^{+}\right\} \text {, } \\
& F_{2}=\operatorname{diag}\left\{\frac{k_{1}^{-}+k_{1}^{+}}{2}, \frac{k_{2}^{-}+k_{2}^{+}}{2}, \cdots, \frac{k_{n}^{-}+k_{n}^{+}}{2}\right\} \\
& h_{21}=h_{2}-h_{1} \text {, } \\
& \tau_{1}=\sum_{l=-h_{2}}^{-h_{1}-1} \sum_{i=l}^{-1} 1=\frac{\left(h_{2}-h_{1}\right)\left(h_{2}+h_{1}+1\right)}{2}, \quad \tau_{2}=\sum_{l=k-h_{2}}^{k-h_{1}-1} \sum_{i=l}^{k-h_{1}-1} 1=\frac{\left(h_{2}-h_{1}\right)\left(h_{2}-h_{1}+1\right)}{2} \\
& \varepsilon_{1}=\sum_{j=-h_{1}}^{-1} \gamma^{-j-1}=\left\{\begin{array}{ll}
h_{1}, & \gamma=1 \\
\frac{\gamma^{h_{1}}-1}{\gamma-1}, & \gamma \neq 1
\end{array}, \quad \varepsilon_{2}=\sum_{j=-h_{2}}^{-1} \gamma^{-j-1}=\left\{\begin{array}{ll}
h_{2}, & \gamma=1 \\
\frac{\gamma^{h_{2}}-1}{\gamma-1}, & \gamma \neq 1
\end{array},\right.\right. \\
& \varepsilon_{3}=\sum_{i=-h_{2}+1}^{-h_{1}} \sum_{j=i}^{-1} \gamma^{-j-1}=\left\{\begin{array}{ll}
\frac{\left(h_{2}-h_{1}\right)\left(h_{2}+h_{1}-1\right)}{2}, & \gamma=1 \\
\frac{\gamma^{h_{2}}-\gamma^{h_{1}}-\left(h_{2}-h_{1}\right)(\gamma-1)}{(\gamma-1)^{2}}, & \gamma \neq 1
\end{array},\right. \\
& \varepsilon_{4}=\sum_{i=-h_{2}}^{-h_{1}-1} \sum_{j=i}^{-1} \gamma^{-j-1}= \begin{cases}\frac{\left(h_{2}-h_{1}\right)\left(h_{2}+h_{1}+1\right)}{2}, & \gamma=1 \\
\frac{\gamma^{h_{2}+1}-\gamma^{h_{1}+1}-\left(h_{2}-h_{1}\right)(\gamma-1)}{(\gamma-1)^{2}}, & \gamma \neq 1\end{cases}
\end{aligned}
$$




$$
\begin{aligned}
& \varepsilon_{5}=\sum_{l=-h_{2}}^{-h_{1}-1} \sum_{i=l}^{-1} \sum_{j=i}^{-1} \gamma^{-j-1} \\
& \varepsilon_{6}=\sum_{l=-h_{2}}^{-h_{1}-1} \sum_{i=l}^{-h_{1}-1} \sum_{j=i}^{-1} \gamma^{-j-1}
\end{aligned}
$$

Proof. Define the following LKF for DNN (1):

$$
V(k)=\sum_{i=1}^{6} V_{i}(k)
$$

with

$$
\begin{aligned}
& V_{1}(k)=x^{T}(k) P x(k) \\
& V_{2}(k)=\sum_{j=k-h_{1}}^{k-1} \gamma^{k-j-1} x^{T}(j) Q_{1} x(j)+\sum_{j=k-h_{2}}^{k-1} \gamma^{k-j-1} x^{T}(j) Q_{2} x(j) \\
& V_{3}(k)=\sum_{j=k-h(k)}^{k-1} \gamma^{k-j-1} x^{T}(j) Q_{3} x(j)+\sum_{i=-h_{2}+1} \sum_{j=k+i}^{k-1} \gamma^{k-j-1} x^{T}(j) Q_{3} x(j) \\
& V_{4}(k)=\sum_{j=k-h(k)}^{k-1} \gamma^{k-j-1} f^{T}(x(j)) R f(x(j))+\sum_{i=-h_{2}+1}^{-h_{1}} \sum_{j=k+i}^{k-1} \gamma^{k-j-1} f^{T}(x(j)) R f(x(j)) \\
& V_{5}(k)=\pi \sum_{i=-h_{2}}^{-h_{1}-1} \sum_{j=k+i}^{k-1} \gamma^{k-j-1} \eta^{T}(j) Z_{1} \eta(j)+\kappa \sum_{l=-h_{2}}^{-h_{1}-1} \sum_{i=l}^{-1} \sum_{j=k+i}^{k-1} \gamma^{k-j-1} \eta^{T}(j) Z_{2} \eta(j) \\
& V_{6}(k)=\vartheta \sum_{l=k-h_{2}}^{k-h_{1}-1} \sum_{i=l}^{k-h_{1}-1} \sum_{j=i}^{k-1} \gamma^{k-j-1} \eta^{T}(j) Z_{3} \eta(j)
\end{aligned}
$$

where $\eta(k)=x(k+1)-x(k)$, and $\pi, \kappa$ and $\vartheta$ are defined in (20). Calculating the difference of LKF (31), $\Delta V(k)=V(k+1)-V(k)$, along the trajectories of DNN (1) gives

$$
\begin{aligned}
\Delta V_{1}(k)= & (\gamma-1) V_{1}+x^{T}(k)\left(C^{T} P C-\gamma P\right) x(k)+2 x^{T}(k) C^{T} P A f(x(k)) \\
& +2 x^{T}(k) C^{T} P A_{d} f(x(k-h(k))) \\
& +f^{T}(x(k)) A^{T} P A f(x(k))+2 f^{T}(x(k)) A^{T} P A_{d} f(x(k-h(k))) \\
& +f^{T}(x(k-h(k))) A_{d}^{T} P A_{d} f(x(k-h(k))) \\
\Delta V_{2}(k)= & (\gamma-1) V_{2}(k)+x^{T}(k)\left(Q_{1}+Q_{2}\right) x(k)-\gamma^{h_{1}} x^{T}\left(k-h_{1}\right) Q_{1} x\left(k-h_{1}\right) \\
& \quad-\gamma^{h_{2}} x^{T}\left(k-h_{2}\right) Q_{2} x\left(k-h_{2}\right)
\end{aligned}
$$




$$
\begin{aligned}
& \Delta V_{3}(k)=\sum_{j=k+1-h(k+1)}^{k} \gamma^{k-j} x^{T}(j) Q_{3} x(j)-\sum_{j=k-h(k)}^{k-1} \gamma^{k-j-1} x^{T}(j) Q_{3} x(j) \\
& +\sum_{i=-h_{2}+1}^{-h_{1}} \sum_{j=k+1+i}^{k} \gamma^{k-j} x^{T}(j) Q_{3} x(j)-\sum_{i=-h_{2}+1}^{-h_{1}} \sum_{j=k+i}^{k-1} \gamma^{k-j-1} x^{T}(j) Q_{3} x(j) \\
& \leq(\gamma-1) V_{3}(k)+\left(h_{2}-h_{1}+1\right) x^{T}(k) Q_{3} x(k)-\gamma^{h_{1}} x^{T}(k-h(k)) Q_{3} x(k-h(k)) \\
& \Delta V_{4}(k) \leq(\gamma-1) V_{4}(k)+\left(h_{2}-h_{1}+1\right) f^{T}(x(k)) R f(x(k)) \\
& -\gamma^{h_{1}} f^{T}(x(k-h(k))) R f(x(k-h(k))) \\
& \Delta V_{5}(k)=(\gamma-1) V_{5}(k)+\eta^{T}(k) Z_{12} \eta(k)-\pi \sum_{j=k-h_{2}}^{k-h_{1}-1} \gamma^{k-j} \eta^{T}(j) Z_{1} \eta(j) \\
& -\kappa \sum_{i=-h_{2}}^{-h_{1}-1} \sum_{j=k+i}^{k-1} \gamma^{k-j} \eta^{T}(j) Z_{2} \eta(j) \\
& \Delta V_{6}(k)=(\gamma-1) V_{6}(k)+\vartheta \tau_{2} \eta^{T}(k) Z_{3} \eta(k)-\vartheta \sum_{l=k-h_{2}}^{k-h_{1}-1} \sum_{i=l}^{k-h_{1}-1} \gamma^{k-i} \eta^{T}(i) Z_{3} \eta(i)
\end{aligned}
$$

where $\tau_{1}, \tau_{2}$ and $Z_{12}$ are defined in (84) and (87).

Based on Lemma 1, we have

$$
\begin{aligned}
& \pi \sum_{j=k-h_{2}}^{k-h_{1}-1} \gamma^{k-j} \eta^{T}(j) Z_{1} \eta(j)=\pi \sum_{j=k-h_{2}}^{k-h(k)-1} \gamma^{k-j} \eta^{T}(j) Z_{1} \eta(j)+\pi \sum_{j=k-h(k)}^{k-h_{1}-1} \gamma^{k-j} \eta^{T}(j) Z_{1} \eta(j) \\
& \geq \frac{1}{\frac{\pi_{1}(k)}{\pi}}\left(\sum_{j=k-h_{2}}^{k-h(k)-1} \eta(j)\right)^{T} Z_{1}\left(\sum_{j=k-h_{2}}^{k-h(k)-1} \eta(j)\right)+\frac{1}{\frac{\pi_{2}(k)}{\pi}}\left(\sum_{j=k-h(k)}^{k-h_{1}-1} \eta(j)\right)^{T} Z_{1}\left(\sum_{j=k-h(k)}^{k-h_{1}-1} \eta(j)\right)
\end{aligned}
$$

where

$$
\begin{aligned}
& \pi_{1}(k)=\sum_{j=k-h_{2}}^{k-h(k)-1} \gamma^{-(k-j)}=\left\{\begin{array}{ll}
h_{2}-h(k), & \gamma=1 \\
\frac{\gamma^{-h(k)}-\gamma^{-h_{2}}}{\gamma-1}, & \gamma \neq 1
\end{array},\right. \\
& \pi_{2}(k)=\sum_{j=k-h(k)}^{k-h_{1}-1} \gamma^{-(k-j)}=\left\{\begin{array}{ll}
h(k)-h_{1}, & \gamma=1 \\
\frac{\gamma^{-h_{1}}-\gamma^{-h(k)}}{\gamma-1}, & \gamma \neq 1
\end{array},\right. \\
& \frac{\pi_{1}(k)}{\pi}+\frac{\pi_{2}(k)}{\pi}=1,
\end{aligned}
$$

For $\left[\begin{array}{cc}Z_{1} & V \\ * & Z_{1}\end{array}\right]>0$, using Lemma 2 we obtain 


$$
\begin{aligned}
\pi \sum_{j=k-h_{2}}^{k-h_{1}-1} \gamma^{k-j} \eta^{T}(j) Z_{1} \eta(j) \geq\left[\begin{array}{c}
\sum_{j=k-h_{2}}^{k-h(k)-1} \eta(j) \\
\sum_{j=k-h(k)}^{k-h_{1}-1} \eta(j)
\end{array}\right]^{T}\left[\begin{array}{cc}
Z_{1} & V \\
* & Z_{1}
\end{array}\right]\left[\begin{array}{c}
\sum_{j=k-h_{2}}^{k-h(k)-1} \eta(j) \\
\sum_{j=k-h(k)}^{k-h_{1}-1}
\end{array}\right] \\
=\left[\begin{array}{c}
x\left(k-h_{1}\right) \\
x(k-h(k)) \\
x\left(k-h_{2}\right)
\end{array}\right]^{T}\left[\begin{array}{ccc}
Z_{1} & -Z_{1}+V^{T} & -V^{T} \\
* & 2 Z_{1}-V-V^{T} & -Z_{1}+V^{T} \\
* & * & Z_{1}
\end{array}\right]\left[\begin{array}{c}
x\left(k-h_{1}\right) \\
x(k-h(k)) \\
x\left(k-h_{2}\right)
\end{array}\right]
\end{aligned}
$$

Similarly, by applying Lemmas 1 and 2, we can find

$$
\begin{aligned}
\kappa \sum_{i=-h_{2}}^{-h_{1}-1} \sum_{j=k+i}^{k-1} \gamma^{k-j} \eta^{T}(j) Z_{2} \eta(j)=\kappa \sum_{i=-h_{2}}^{-h(k)-1} \sum_{j=k+i}^{k-1} \gamma^{k-j} \eta^{T}(j) Z_{2} \eta(j)+\kappa \sum_{i=-h(k)}^{-h_{1}-1} \sum_{j=k+i}^{k-1} \gamma^{k-j} \eta^{T}(j) Z_{2} \eta(j) \\
\geq \\
\geq\left[\begin{array}{c}
x(k) \\
\phi(k) \\
\varphi(k)
\end{array}\right]^{T}\left[\begin{array}{ccc}
a(h(k)) I_{n} & -I_{n} & 0_{n} \\
b(h(k)) I_{n} & 0_{n} & -I_{n}
\end{array}\right]^{T}\left[\begin{array}{cc}
Z_{2} & U \\
* & Z_{2}
\end{array}\right]\left[\begin{array}{lll}
a(h(k)) I_{n} & -I_{n} & 0_{n} \\
b(h(k)) I_{n} & 0_{n} & -I_{n}
\end{array}\right]\left[\begin{array}{c}
x(k) \\
\phi(k) \\
\varphi(k)
\end{array}\right] \\
=\xi^{T}(k) \Gamma^{T}(h(k))\left[\begin{array}{cc}
Z_{2} & U \\
* & Z_{2}
\end{array}\right] \Gamma(h(k)) \xi(k)
\end{aligned}
$$

where

$$
\begin{aligned}
& a(h(k))=h_{2}-h(k), \quad b(h(k))=h(k)-h_{1}, \\
& \Gamma(h(k))=\left[\begin{array}{llll}
a(h(k)) I_{n} & 0_{n \times 5 n} & -I_{n} & 0_{n} \\
b(h(k)) I_{n} & 0_{n \times 5 n} & 0_{n} & -I_{n}
\end{array}\right], \quad\left[\begin{array}{cc}
Z_{2} & U \\
* & Z_{2}
\end{array}\right]>0 \\
& \phi(k)=\sum_{j=k-h_{2}}^{k-h(k)-1} x(j), \quad \varphi(k)=\sum_{j=k-h(k)}^{k-h_{1}-1} x(j), \\
& \xi(k)=\left[x^{T}(k) x^{T}\left(k-h_{1}\right) x^{T}(k-h(k)) x^{T}\left(k-h_{2}\right) f^{T}(x(k)) f^{T}(x(k-h(k))) \phi^{T}(k) \varphi^{T}(k)\right]^{T}
\end{aligned}
$$

Furthermore, we can obtain

$$
\begin{aligned}
& \vartheta \sum_{l=k-h_{2}}^{k-h_{1}-1} \sum_{i=l}^{k-h_{1}-1} \gamma^{k-i} \eta^{T}(i) Z_{3} \eta(i) \geq\left(\sum_{l=k-h_{2}}^{k-h_{1}-1} \sum_{i=l}^{k-h_{1}-1} \eta(i)\right)^{T} Z_{3}\left(\sum_{l=k-h_{2}}^{k-h_{1}-1} \sum_{i=l}^{k-h_{1}-1} \eta(i)\right) \\
& =\left(\left(h_{2}-h_{1}\right) x\left(k-h_{1}\right)-\sum_{l=k-h_{2}}^{k-h_{1}-1} x(l)\right)^{T} Z_{3}\left(\left(h_{2}-h_{1}\right) x\left(k-h_{1}\right)-\sum_{l=k-h_{2}}^{k-h_{1}-1} x(l)\right) \\
& =\left(h_{21} x\left(k-h_{1}\right)-\phi(k)-\varphi(k)\right)^{T} Z_{3}\left(h_{21} x\left(k-h_{1}\right)-\phi(k)-\varphi(k)\right)
\end{aligned}
$$

Taking the assumption regarding the activation functions (4) into account, we can obtain

$$
\left[\begin{array}{c}
x(k) \\
f(x(k))
\end{array}\right]^{T}\left[\begin{array}{cc}
F_{1} \Lambda_{1} & -F_{2} \Lambda_{1} \\
-F_{2} \Lambda_{1} & \Lambda_{1}
\end{array}\right]\left[\begin{array}{c}
x(k) \\
f(x(k))
\end{array}\right] \leq 0
$$




$$
\left[\begin{array}{c}
x(k-h(k)) \\
f(x(k-h(k)))
\end{array}\right]^{T}\left[\begin{array}{cc}
F_{1} \Lambda_{2} & -F_{2} \Lambda_{2} \\
-F_{2} \Lambda_{2} & \Lambda_{2}
\end{array}\right]\left[\begin{array}{c}
x(k-h(k)) \\
g(x(k-h(k)))
\end{array}\right] \leq 0
$$

where $\Lambda_{i}$ and $F_{i}, i=1,2$ are defined by (86).

By combining the previous inequalities (32)-(99), (102), (103), and (105)-(107) we have

$$
\Delta V(k)-(\gamma-1) V(k) \leq \xi^{T}(k)\left(\Sigma-\Gamma(h(k))\left[\begin{array}{cc}
Z_{2} & U \\
* & Z_{2}
\end{array}\right] \Gamma^{T}(h(k))\right) \xi(k)
$$

where $\Sigma$ is given in (83). If

$$
\Sigma-\Gamma(h(k))\left[\begin{array}{cc}
Z_{2} & U \\
* & Z_{2}
\end{array}\right] \Gamma^{T}(h(k))<0, \quad \forall h(k) \in\left[h_{1}, h_{2}\right]
$$

then

$$
\Delta V(k)-(\gamma-1) V(k)<0
$$

From (109), based on Lemma 3, there exist a matrix $\Omega$ such that

$$
\Phi(h(k))=\left[\begin{array}{ccc}
\Sigma+\Gamma(h(k)) \Omega^{T}+\Omega \Gamma^{T}(h(k)) & \Omega_{1} & \Omega_{2} \\
* & -Z_{2} & -U \\
* & * & -Z_{2}
\end{array}\right]<0, \quad \forall h(k) \in\left[h_{1}, h_{2}\right]
$$

Since $\Phi(h(k))$ is affine with respect to $h(k)$, it is necessary and sufficient to ensure that inequality (111) holds at the vertices of the interval $\left[h_{1}, h_{2}\right]$, i.e. $\Phi\left(h_{1}\right)<0$ and $\Phi\left(h_{2}\right)<0$, where $\Gamma\left(h_{1}\right)=\Gamma_{1}$ and $\Gamma\left(h_{2}\right)=\Gamma_{2}$. Thus, the inequality (111) holds if and only if (79) holds as well.

From (110), it follows

$$
V(k)<\gamma V(k-1)<\gamma^{2} V(k-2)<\cdots<\gamma^{k} V(0), \quad k=1,2,3, \cdots
$$

From (31) and (112), we obtain

$$
\begin{aligned}
& V(0) \leq \alpha \lambda_{\max }(P)+\alpha \varepsilon_{1} \lambda_{\max }\left(Q_{1}\right)+\alpha \varepsilon_{2} \lambda_{\max }\left(Q_{2}\right)+\alpha \varepsilon_{2} \lambda_{\max }\left(Q_{3}\right)+\alpha \varepsilon_{3} \lambda_{\max }\left(Q_{3}\right) \\
& \quad+\hat{f}^{2} \varepsilon_{2} \lambda_{\max }(R)+\hat{f}^{2} \varepsilon_{3} \lambda_{\max }(R)+\pi \delta \varepsilon_{4} \lambda_{\max }\left(Z_{1}\right)+\kappa \delta \varepsilon_{5} \lambda_{\max }\left(Z_{2}\right)+\vartheta \delta \varepsilon_{6} \lambda_{\max }\left(Z_{3}\right)
\end{aligned}
$$

where $\varepsilon_{i}, i=1,2, \cdots, 6$ and $\hat{f}^{2}$ are defined in Theorem 3. On the other hand, we have

$$
V(k) \geq \lambda_{\min }(P) x^{T}(k) x(k)
$$

From (112), (113) and (114), for $\gamma>1$, we can obtain

$$
\begin{aligned}
\lambda_{\min }(P) x^{T}(k) x(k) & \leq \gamma^{N}\left[\alpha \lambda_{\max }(P)+\alpha \varepsilon_{1} \lambda_{\max }\left(Q_{1}\right)+\alpha \varepsilon_{2} \lambda_{\max }\left(Q_{2}\right)+\alpha\left(\varepsilon_{2}+\varepsilon_{3}\right) \lambda_{\max }\left(Q_{3}\right)\right. \\
& \left.+\hat{f}^{2}\left(\varepsilon_{2}+\varepsilon_{3}\right) \lambda_{\max }(R)+\pi \delta \varepsilon_{4} \lambda_{\max }\left(Z_{1}\right)+\kappa \delta \varepsilon_{5} \lambda_{\max }\left(Z_{2}\right)+\vartheta \delta \varepsilon_{6} \lambda_{\max }\left(Z_{3}\right)\right]
\end{aligned}
$$


If

$$
\begin{aligned}
& \frac{\gamma^{N}}{\lambda_{\text {min }}(P)}\left[\alpha \lambda_{\text {max }}(P)+\alpha \varepsilon_{1} \lambda_{\text {max }}\left(Q_{1}\right)+\alpha \varepsilon_{2} \lambda_{\text {max }}\left(Q_{2}\right)+\alpha\left(\varepsilon_{2}+\varepsilon_{3}\right) \lambda_{\text {max }}\left(Q_{3}\right)\right. \\
& \left.\quad+\hat{f}^{2}\left(\varepsilon_{2}+\varepsilon_{3}\right) \lambda_{\text {max }}(R)+\pi \delta \varepsilon_{4} \lambda_{\text {max }}\left(Z_{1}\right)+\kappa \delta \varepsilon_{5} \lambda_{\text {max }}\left(Z_{2}\right)+\vartheta \delta \varepsilon_{6} \lambda_{\text {max }}\left(Z_{3}\right)\right]<\beta
\end{aligned}
$$

then we obtain $x^{T}(k) x(k)<\beta, \forall k \in\{1,2, \ldots, N\}$, and the system (1) is finite-time stable with respect to $(\alpha, \beta, N)$.

Let

$$
\begin{aligned}
& \lambda_{1} I<\lambda_{\text {max }}(P)<\lambda I_{2}, \quad \lambda_{\text {max }}\left(Q_{1}\right)<\lambda_{3}, \quad \lambda_{\text {max }}\left(Q_{2}\right)<\lambda_{4}, \quad \lambda_{\text {max }}\left(Q_{3}\right)<\lambda_{5}, \\
& \lambda_{\text {max }}(R)<\lambda_{6}, \quad \lambda_{\text {max }}\left(Z_{1}\right)<\lambda_{7}, \quad \lambda_{\text {max }}\left(Z_{2}\right)<\lambda_{8}, \quad \lambda_{\text {max }}\left(Z_{3}\right)<\lambda_{9}
\end{aligned}
$$

Then, the conditions (81) and (82) follow from (116) and (117).

Now, we focus on the finite-time passivity of DNN (1) with time-varying delay.

Theorem 4. [16] Under Assumption 1, DNN (1) is finite time passive with respect to $(\alpha, \beta, N, \mu)$, where $0 \leq \alpha<\beta, \mu$ is a given positive scalar if there exist a scalar $\gamma>1$, positive scalars $\lambda_{i}, i=1,2, \ldots, 9$, positive definite matrices $P, Q_{1}, Q_{2}, Q_{3}, R, Z_{1}, Z_{2}$, and $Z_{3}$, positive definite diagonal matrices $\Lambda_{1}$ and $\Lambda_{2}$, matrices $U, V$ and $\Omega=[\Omega 1 \Omega 2]$ such that the LMIs (79)-(82) hold and:

$$
\left[\begin{array}{ccc}
\hat{\Sigma}+\hat{\Gamma}_{i} \Omega^{T}+\Omega \hat{\Gamma}_{i}^{T} & \Omega_{1} & \Omega_{2} \\
* & -Z_{2} & -U \\
* & * & -Z_{2}
\end{array}\right]<0, \quad i=1,2
$$

where

$$
\begin{aligned}
& \hat{\Sigma}=\left[\begin{array}{cc}
\Sigma & \Upsilon \\
\Upsilon^{T} & -\mu I_{n}
\end{array}\right], \quad \Upsilon=\left[\begin{array}{c}
0_{4 n \times n} \\
-I_{n} \\
0_{3 n \times n}
\end{array}\right] \in \mathfrak{R}^{8 n \times n} \\
& \hat{\Gamma}_{1}=\left[\begin{array}{c}
\Gamma_{1} \\
0_{n \times 2 n}
\end{array}\right], \quad \hat{\Gamma}_{2}=\left[\begin{array}{c}
\Gamma_{2} \\
0_{n \times 2 n}
\end{array}\right]
\end{aligned}
$$

in which $\Sigma, \Gamma_{1}$ and $\Gamma_{2}$ are defined as in (83) and (84).

Proof. To show the passivity, we chose LKF (31). Then, from the proof of Theorem 3, we can write

$$
\begin{aligned}
\Delta V(k)- & (\gamma-1) V(k)-2 y^{T}(k) u(k)-\mu u^{T}(k) u(k) \\
& \leq \xi^{T}(k)\left(\Sigma-\Gamma(h(k))\left[\begin{array}{cc}
Z_{2} & U \\
* & Z_{2}
\end{array}\right] \Gamma^{T}(h(k))\right) \xi(k)-2 f^{T}(x(k)) u(k)-\mu u^{T}(k) u(k) \\
& =\hat{\xi}^{T}(k)\left(\hat{\Sigma}-\hat{\Gamma}(h(k))\left[\begin{array}{cc}
Z_{2} & U \\
* & Z_{2}
\end{array}\right] \hat{\Gamma}^{T}(h(k))\right) \hat{\xi}(k)
\end{aligned}
$$


where

$$
\hat{\xi}(k)=\left[\xi^{T}(k) u^{T}(k)\right]^{T}, \quad \hat{\Gamma}(h(k))=\left[\begin{array}{c}
\Gamma(h(k)) \\
0_{n \times 2 n}
\end{array}\right]
$$

and $\hat{\Sigma}$ is defined by (119). If

$$
\hat{\Sigma}-\hat{\Gamma}(h(k))\left[\begin{array}{cc}
Z_{2} & U \\
* & Z_{2}
\end{array}\right] \hat{\Gamma}^{T}(h(k))<0, \quad \forall h(k) \in\left[h_{1}, h_{2}\right]
$$

then

$$
\Delta V(k)-(\gamma-1) V(k)-2 y^{T}(k) u(k)-\mu u^{T}(k) u(k)<0
$$

Based on Lemma 3, then from (123) we obtain

$$
\hat{\Phi}(h(k))=\left[\begin{array}{ccc}
\hat{\Sigma}+\hat{\Gamma}(h(k)) \Omega^{T}+\Omega \hat{\Gamma}^{T}(h(k)) & \Omega_{1} & \Omega_{2} \\
* & -Z_{2} & -U \\
* & * & -Z_{2}
\end{array}\right]<0, \quad \forall h(k) \in\left[h_{1}, h_{2}\right]
$$

Since $\hat{\Phi}(h(k))$ is affine with respect to $h(k)$, it is necessary and sufficient to ensure that inequality (125) holds at the vertices of the interval $\left[h_{1}, h_{2}\right]$, i.e. $\hat{\Phi}\left(h_{1}\right)<0$ and $\hat{\Phi}\left(h_{2}\right)<0$, where $\hat{\Gamma}\left(h_{1}\right)=\hat{\Gamma}_{1}$ and $\hat{\Gamma}\left(h_{2}\right)=\hat{\Gamma}_{2}$. Thus, the inequality (125) holds if and only if (118) holds as well.

From (124), we get

$$
0<V(k)<\gamma^{k} V(0)+2 \sum_{j=0}^{k-1} \gamma^{k-j-1} y^{T}(j) u(j)+\mu \sum_{j=0}^{k-1} \gamma^{k-j-1} u^{T}(j) u(j)
$$

Under the zero initial condition, for $k \in\{1,2, \cdots, N\}$ one has

$$
2 \sum_{j=0}^{k-1} \gamma^{k-j-1} y^{T}(j) u(j)+\mu \sum_{j=0}^{k-1} \gamma^{k-j-1} u^{T}(j) u(j) \geq 0
$$

i.e.

$$
2 \sum_{j=0}^{k-1} \gamma^{-j} y^{T}(j) u(j) \geq-\mu \sum_{j=0}^{k-1} \gamma^{-j} u^{T}(j) u(j) \geq-\mu \sum_{j=0}^{k-1} u^{T}(j) u(j)
$$

Thus, the system (1) is finite-time passive.

Remark 8. In [16], the properties of exponential passivity (Definition 2) and finite-time passivity (Theorem 4) for DNNs with time-varying delay are introduced for the first time, in which FTP is based on the concept of FTS (Theorem 3). Since FTS in one part uses the property of exponential stability (see inequality (110)), then in the proof of Theorem 4, the condition (15) has appeared as expected. Unlike the concept of FTP, a well-known concept for the passivity based on Lyapunov asymptotic stability has already been reported in the literature. Note that a sufficient condition for the passivity of DNNs can be obtained when $\gamma=1$ in Theorem 4 .

Remark 9. Using Lemma 3, inequalities (79) and (118) can be transformed in the following equivalent forms 


$$
\begin{aligned}
& \Sigma<\Gamma_{1}\left[\begin{array}{cc}
Z_{2} & U \\
U^{T} & Z_{2}
\end{array}\right] \Gamma_{1}^{T}=\left[\begin{array}{cccc}
h_{21}^{2} Z_{2} & 0_{n \times 5 n} & -h_{21} Z_{2} & -h_{21} U \\
* & 0_{5 n \times 5 n} & 0_{5 n \times n} & 0_{5 n \times n} \\
* & * & Z_{2} & U \\
* & * & * & Z_{2}
\end{array}\right]=\Xi_{1} \\
& \Sigma<\Gamma_{2}\left[\begin{array}{cc}
Z_{2} & U \\
U^{T} & Z_{2}
\end{array}\right] \Gamma_{2}^{T}=\left[\begin{array}{cccc}
h_{21}^{2} Z_{2} & 0_{n \times 5 n} & -h_{21} U^{T} & -h_{21} Z_{2} \\
* & 0_{5 n \times 5 n} & 0_{5 n \times n} & 0_{5 n \times n} \\
* & * & Z_{2} & U \\
* & * & * & Z_{2}
\end{array}\right]=\Xi_{2} \\
& \hat{\Sigma}<\hat{\Gamma}_{1}\left[\begin{array}{cc}
Z_{2} & U \\
* & Z_{2}
\end{array}\right] \hat{\Gamma}_{1}^{T}=\left[\begin{array}{c:c}
\Xi_{1} & 0_{8 n \times n} \\
* & 0_{n}
\end{array}\right] \\
& \hat{\Sigma}<\hat{\Gamma}_{2}\left[\begin{array}{cc}
Z_{2} & U \\
* & Z_{2}
\end{array}\right] \hat{\Gamma}_{2}^{T}=\left[\begin{array}{c:c}
\Xi_{2} & 0_{8 n \times n} \\
* & 0_{n}
\end{array}\right]
\end{aligned}
$$

The above inequalities will be used in numerical calculations.

Remark 10. The proposed stability criteria depend on the parameters $\gamma, \alpha, \beta, N$ as well as time delay $h_{1}$ and $h_{2}$. Consider the following cases.

- $\beta_{\min }$ is the minimum allowable lower bound (MALB) of $\beta$ such that the concerned DNN is FTS (FTP) for any $\beta \geq \beta_{\min }$.

- $h_{\max }=h_{2 \max }$ is the maximum allowable upper bound (MAUB) of the variable delay $h(k)$ such that the concerned DNN is FTS (FTP) for any value when the delay $\leq h_{\max }$. Note that a criterion that gives a lower MALB value or a higher MAUB value is less conservative with respect to the other criteria.

Remark 11. In [16], the criteria of FTS are defined with respect to the adjustable parameter $\gamma$ and the new LKF $V(\psi)$ with power function $\gamma^{\psi-j-1}$ is proposed. From the difference of LKF given in (52), $\Delta V(\psi)<(\gamma-1) V(\psi)$, it follows that the parameter $\gamma$ provides an exponential convergence information about an upper bound of LKF given in (54), $V(\psi)<\gamma^{\psi} V(0), \quad \psi=1,2,3, \cdots$. On the other hand, parameterized condition (6) with power function $\gamma^{-j}$ is obtained while analyzing the FTP criterion. This condition can be understood as a condition of exponential passivity with adjustable parameter $\gamma$. In the case of $\gamma=1$, the condition (6) reduces to the ordinary passivity defined in the existing literature. The parameter $\gamma$ represent an optimization parameter in the FTP criterion. By solving the corresponding LMIs for FTP, a specific value for $\gamma$ is obtained and the exponential passivity condition (6) is satisfied.

Remark 12. The results proposed in [16] are delay-dependent, but do not depend on the shape of the time delay. Therefore, the delay may be a random variable. In the case of switching systems, the considered FTP problem can also be solved. In this case, it is necessary to modify the Lyapunov functional in accordance with the given switching systems. 


\section{DEMONSTRATIVE EXAMPLES}

In the section, three numerical examples $[16,56]$ are provided to illustrate the effectiveness of the proposed stability criteria.

Example 1. Consider the DNN (1) with the following parameters [56]

$$
C=\left[\begin{array}{cc}
0.8 & 0 \\
0 & 0.9
\end{array}\right], \quad A=\left[\begin{array}{cc}
0.001 & 0 \\
0 & 0.005
\end{array}\right], \quad A_{d}=\left[\begin{array}{cc}
-0.1 & 0.01 \\
-0.2 & -0.1
\end{array}\right]
$$

which was used to check the feasible region of stability criteria in $[24,31,56,57,58]$. The activation function is in the form of $f(x(k))=\left[\tanh \left(x_{1}(k)\right) \tanh \left(x_{2}(k)\right)\right]^{T}$ and satisfy Assumption 1 with $k_{1}^{-}=k_{2}^{-}=0$ and $k_{1}^{+}=k_{2}^{+}=1$. By using Theorem 1, the MAUBs of $h_{2 \max }$ are computed with different lover bounds $h_{1}$ and the obtained results are given in Tab. 1. The number of decision variables (NDVs) is also given to show computation complexity. From this table, we can see that proposed results are less conservative and/or have less decision variables than $[24,31,57,58]$.

The time-varying delay and the state trajectories of the DNN (1) with parameters (133) for initial value $\phi(j)=[31]^{T}, j \in\{-22,-21, \ldots,-1,0\}$ are shown in Fig. 1 and Fig. 2, which shows that the DNN (1) is asymptotically stable for $10 \leq h(k) \leq 22$.

Table 1 MAUBs of $h_{2 \max }$ for a given $h_{1}$ in Example 1 .

\begin{tabular}{ccccccccc}
\hline Method & \multicolumn{7}{c}{$h_{1}$} & \multirow{2}{*}{ NDVs } \\
\cline { 2 - 7 } & 2 & 4 & 6 & 8 & 10 & 15 & 20 & \\
\hline Th. 2 [57] & 19 & 19 & 20 & 21 & 22 & 24 & 27 & $17.5 n^{2}+8.5 n$ \\
Th. 1 [57] & 20 & 20 & 21 & 21 & 22 & 24 & 27 & $53.5 n^{2}+8.5 n$ \\
Th. 1 [31] & - & 20 & 20 & 21 & 22 & 24 & - & $13.5 n^{2}+11.5 n$ \\
Th. 3.1 [24] & 19 & 19 & 20 & 20 & 21 & 24 & 27 & $11 n^{2}+6 n$ \\
Cor. 2 [58] & 19 & 20 & 20 & 21 & 21 & 24 & 27 & $154 n^{2}+6 n$ \\
\hline Theorem 1 & 19 & 20 & 20 & 21 & 22 & 24 & 27 & $17 n^{2}+9 n$ \\
\hline Theorem 2 & 20 & 20 & 21 & 21 & 22 & 24 & 27 & $23 n^{2}+11 n$ \\
\hline
\end{tabular}

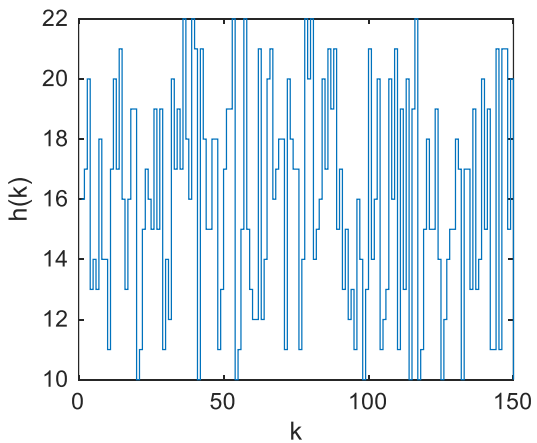

Fig. 1 The time-varying delay

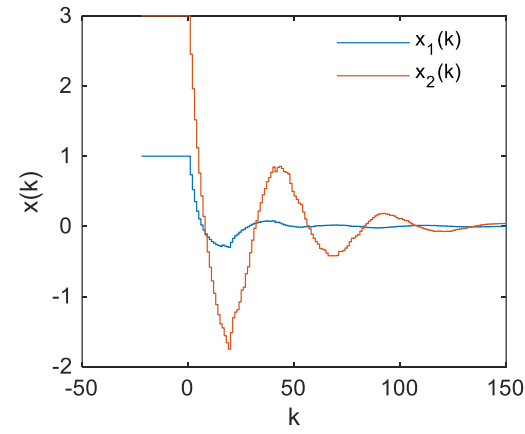

Fig. 2 State trajectories of the DNN 
Example 2. Consider DNN (1) with the following parameters [31], [56]

$$
C=\left[\begin{array}{cc}
0.1 & 0 \\
0 & 0.3
\end{array}\right], \quad A=\left[\begin{array}{cc}
0.02 & 0 \\
0 & 0.004
\end{array}\right], \quad A_{d}=\left[\begin{array}{cc}
-0.01 & 0.01 \\
-0.02 & -0.01
\end{array}\right]
$$

and activation functions that satisfy Assumption 1 with $k_{1}^{-}=k_{2}^{-}=0$ and $k_{1}^{+}=k_{2}^{+}=1$. In this example, the MAUBs of $h_{2 \max }$ are computed by using Theorem 1 and 2 for different lover bounds $h_{1}$ and results are shown in Tab. 2. From this table, it can be seen that the proposed stability criteria in Theorems 1 and 2, provide a significantly larger MAUB than the previous result [31], while the numbers of decision variables are slightly higher.

Table 2 MAUBs of $h_{2 \max }$ for a given $h_{1}$ in Example 2.

\begin{tabular}{|c|c|c|c|c|c|c|c|}
\hline \multirow{2}{*}{ Method } & \multicolumn{6}{|c|}{$h_{1}$} & \multirow{2}{*}{ NDVs } \\
\hline & 2 & 4 & 6 & 8 & 10 & 20 & \\
\hline Th. 1 [31] & 99 & 101 & 103 & 105 & 107 & 117 & $13.5 n^{2}+11.5 n$ \\
\hline Theorem 1 & 2028 & 2030 & 2032 & 2034 & 2036 & 2046 & $17 n^{2}+9 n$ \\
\hline Theorem 2 & 3140 & 3142 & 3144 & 3146 & 3148 & 3158 & $23 n^{2}+11 n$ \\
\hline
\end{tabular}

Example 3. Consider DNN (1) with the following parameters [16], [48]

$$
C=\left[\begin{array}{cc}
0.6 & 0 \\
0 & 0.4
\end{array}\right], \quad A=\left[\begin{array}{cc}
-0.1 & 0.8 \\
0.9 & 0.4
\end{array}\right], \quad A_{d}=\left[\begin{array}{cc}
-0.2 & -0.9 \\
0.1 & 0.2
\end{array}\right]
$$

The activation functions are described by $f(x(k))=\left[\tanh \left(0.2 x_{1}(k)\right) \tanh \left(-0.14 x_{2}(k)\right)\right]^{T}$. Therefore, it is easy to see that $F_{1}=\operatorname{diag}\{0,0\}$ and $F_{2}=\operatorname{diag}\{0.1,-0.07\}$.

First, the FTS of DNN (1) is investigated. Let $h_{1}=1$ and $h_{2}=3$, then by solving LMIs in Theorem 3 for $\alpha=1, \beta=2, N=30, \delta=2.5$ and $\gamma=1.01$ we obtain the feasible solutions. Thus, the system (1) with parameters (133) is finite-time stable with respect to $(1,2,30)$. For time-varying delay $h(k)=2+\sin (k \pi / 2)$ and the initial condition $\left[\begin{array}{lll}-0.5 & 0.5\end{array}\right]^{T}$ Fig. 3 shows the state response $x(k)$ of DNN (1) with the parameters (133) and Fig. 4 depicts the norm $x^{T}(k) x(k)$ of the state vector of the system (1).

Furthermore, by using Theorem 3, MALBs of $\beta_{\text {min }}$ are calculated for $\alpha=1, N=30$, $\delta=2.5, h_{1}=1, \gamma=1.0001$ and $h_{2} \in\{3,4,5,8,10\}$, and results are listed in Tab. 3 .

Table 3 MALBs of $\beta_{\min }$ for a given $h_{2}$ when $\alpha=1, \delta=2.5, h_{1}=1, N=30$ and $\gamma=1.0001$ in Example 3

\begin{tabular}{lcccccc}
\hline$h_{2}$ & 3 & 4 & 5 & 8 & 10 & 20 \\
\hline Theorem 3 & 1.19 & 1.32 & 1.49 & 2.23 & 2.96 & 10.40 \\
\hline
\end{tabular}

Based on Fig. 4, we can see that the norm $x^{T}(k) x(k)$ does not exceed MALB $\beta_{\min }$ in Tab. 3 , which means that the above system is FTS with respect to $\left(3, \beta_{\min }, N\right), N \in(5,10,20,40)$.

For different values of $N \in(50,100,150,200)$ MAUBs of $h_{\max }$ are computed for $\alpha=1$, $\beta=1000, \delta=2.5$ and results are shown in Tab. 4. These results are mutually equal because the considered system is asymptotic stable $\left(\gamma \rightarrow 1 \Rightarrow \gamma^{N} \rightarrow 1\right)$, so the inequality (82) becomes insensitive to the parameter $N$. 
Table 4 MAUBs of $h_{\max }$ for a given $N$ when $h_{1}=5, \alpha=1, \beta=10$ and $\gamma=1.0001$ in Example 3

\begin{tabular}{lccccc}
\hline$N$ & 50 & 100 & 150 & 200 & 500 \\
\hline Theorem 3 & 20 & 20 & 20 & 20 & 20 \\
\hline
\end{tabular}

Second, we consider FTP of DNN (1) with parameters (133). By applying Theorem 4, we can obtain MALBs of $\beta_{\text {min }}$ for $\alpha=1, \delta=2.5, N=30, h_{1}=1$ and $\mu$ for given $h_{2}$, which guarantees DNN (1) with parameters (133) is FTP in the sense of Definition 2. In addition, FTP of DNN (1) with parameters (133) is analyzed by using the same method in [48]. The obtained results are addressed in Tab. 5, which demonstrates that this approach provides less conservative results than the approach in [48].

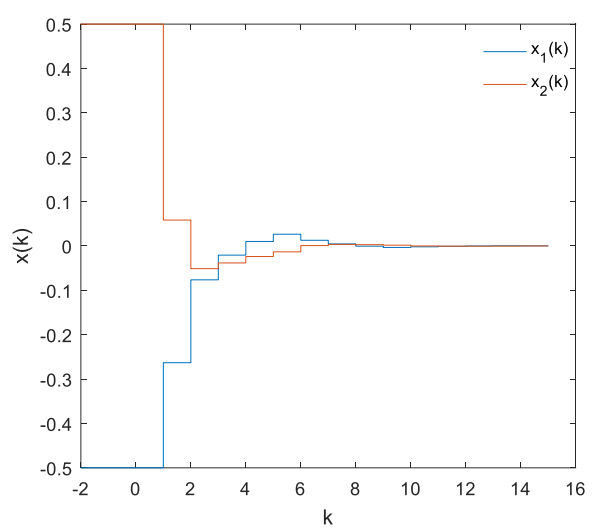

Fig. 3 The state response $x(k)$ of the system (1) for $k>0$ and the initial conditions $\phi(k)$ for $k<0$.

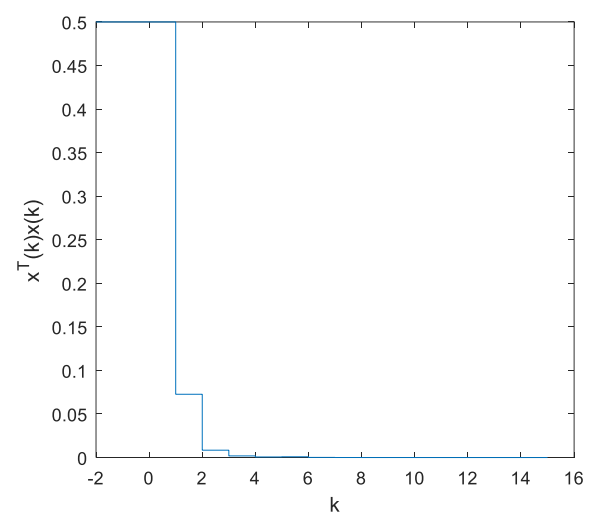

Fig. 4 The norm $x^{T}(k) x(k)$ of the system (1) for $k>0$ and the norm of the initial conditions $\phi^{T}(k) \phi(k)$ for $k<0$.

Table 5 MALBs of $\beta_{\min }$ for a given $h_{2}$ when $\alpha=1, \delta=2.5, N=30$ and $\gamma=1.02$ in Example 3.

\begin{tabular}{lccccc}
\hline$h_{2}$ & 3 & 4 & 5 & 8 & 10 \\
\hline$[48]$ & 4.09 & 4.67 & 5.34 & 7.18 & 9.95 \\
\hline Theorem 4 & $2.15 /$ & $2.38 /$ & $2.67 /$ & $4.02 /$ & $5.38 /$ \\
$\beta_{\min } / \mu$ & 8.90 & 2.82 & 14.22 & 72.30 & 36.19 \\
\hline
\end{tabular}

In order to check the conditions of passivity, the following scalar is defined

$$
\mathrm{CP}=2 \sum_{j=0}^{\psi-1} \gamma^{-j} y^{T}(j) u(j)+\mu \sum_{j=0}^{\psi-1} u^{T}(j) u(j), \quad \forall \psi \in\{1,2, \cdots, N\}
$$

Based on (15), the system will be passive if $\mathrm{CP} \geq 0$. By using results of Theorem 4, the values of $\mathrm{CP}$ are computed from (136) for $\alpha=1, \delta=2.5, N=30, \gamma=1.02$ and given $h_{2}$ and listed in Tab. 6. Since the values of $\mathrm{CP}$ are positive, it can be concluded that the passivity condition (15) is satisfied. 
Table 6 Checking the passivity condition (15) by using the scalar CP defined in (136) for $\alpha=1, \delta=2.5, N=30, \gamma=1.02$ and given $h_{2}$ in Example 3 .

\begin{tabular}{lccccc}
\hline$h_{2}$ & 3 & 4 & 5 & 8 & 10 \\
\hline $\mathrm{CP}$ & 69.25 & 23.11 & 109.55 & 549.79 & 276.06 \\
\hline
\end{tabular}

Remark 13. To reduce the number decision variables, the well-known reciprocally convex combination approach is used in [16]. It is noted that, the addition of slack variables U1, U2, U3 and U4 in [48] will increase the number of decision variables. The number of decision variables in [48] is $15 n^{2} / 2+11 n / 2+10$, while the number of decision variable of Theorem 4 is slightly smaller and amounts to $6 n^{2}+6 n+10$.

Remark 14. From Example 3 and Remark 13 it can be concluded that the results in [16] are less conservative then results proposed in [48]. According to the author's knowledge, no other references, except [48], deal with the problem of FTP for delayed DNNs. Other references in the literature of [16] consider different stability concepts from that used in [16].

\section{CONCLUSION}

This paper deals with the stability, finite-time stability and passivity problems of delayed DNNs [16, 56]. First, the Lyapunov-Krasovskii functional with single and double summation terms and several augmented vectors is constructed by decomposing the time-delay interval into two non-equidistant subintervals. Using the Wirtinger-based inequality, extended reciprocally convex approach and several zero equalities, stability conditions are developed in the form of linear matrix inequalities. Second, a delay-dependent criterion has been established to ensure that the considered DNN is passive. By constructing the proper LKF and using LMI techniques, sufficient passivity conditions are obtained. Moreover, some weighted summation inequalities are proposed to obtain less conservative results. Finally, several numerical examples are presented to show the effectiveness of the proposed methods.

Acknowledgement: This work has been supported by the Ministry of Education, Science and Technological Development of the Republic of Serbia, Program for financing scientific research work, ev. no. 451-03-68/2020-14/200133.

\section{REFERENCES}

[1] Y.H. Chen, and S.C. Fang, „Neurocomputing with time delay analysis for solving convex quadratic programming problems" IEEE Transactions on Neural Networks, vol. 11, no. 1, pp. 230-240, 2000

[2] L. Zhang, and Z. Yi, ,Selectable and unselectable sets of neurons in recurrent neural networks with saturated piecewise linear transfer function”, IEEE Transactions on Neural Networks, vol. 22, no. 7, pp. 1021-1031, 2011.

[3] Y. Liu, Z. Wang, and X. Liu, „Global exponential stability of generalized recurrent neural networks with discrete and distributed delays," Neural Networks, vol. 19, no. 5, pp. 667-675, 2006.

[4] J. Sun, G. P. Liu, J. Chen, and D. Rees, „Improved delay-range dependent stability criteria for linear systems with time-varying delays," Automatica, vol. 46, no. 2, pp. 466-470, 2010.

[5] O.M. Kwon, M.J. Park, J.H. Park, S.M. Lee, and E.J. Cha, „Improved delay-partitioning approach to robust stability analysis for discrete-time systems with time-varying delays and randomly occurring parameter uncertainties” Optimal Control Applications and Methods, vol. 36, no. 4, pp. 496-511, 2015.

[6] Y. He, M.D. Ji, C.K. Zhang, and M. Wu, ,Global exponential stability of neural networks with time-varying delay based on free-matrix-based integral inequality" Neural Networks, vol. 77, pp. 80-86, 2016. 
[7] H. Chen, S. Zhong, and J. Yang, „A new globally exponential stability criterion for neural networks with discrete and distributed delays" Mathematical Problems in Engineering, vol. 2015, Article ID 807150, 9 pages, 2015 .

[8] S.-P. Xiao, H.-H. Lian, H.-B. Zeng, G. Chen, and W.-H. Zheng, „Analysis on Robust Passivity of Uncertain Neural Networks with Time-varying Delays via Free-matrix-based Integral Inequality", International Journal of Control, Automation and Systems, vol. 15, no. 5, pp. 2385-2394, 2017.

[9] X.-M. Zhang, Q.-L. Han, X. Ge, and D. Ding, „An overview of recent developments in LyapunovKrasovskii functionals and stability criteria for recurrent neural networks with time-varying delays", Neurocomputing, vol. 313, pp. 392-401, 2018.

[10] H. Zhang, Z. Wang and D. Liu, ,A Comprehensive Review of Stability Analysis of Continuous-Time Recurrent Neural Networks“, IEEE Transactions on Neural Networks and Learning Systems, vol. 25, no. 7, pp. 1229-1262, 2014

[11] C.W. Song, H.J. Gao, and W.X. Zheng, „A new approach to stability analysis of discrete-time recurrent neural networks with time-varying delay", Neurocomputing, vol. 72 no. 10-12, pp. 2563-2568, 2009.

[12] D. Zhang, L. Yu, Q.G. Wang, and C.J. Ong, ,Estimator design for discrete-time switched neural networks with asynchronous switching and time-varying delay" IEEE Transactions on Neural Networks and Learning Systems, vol. 23, no. 5, pp. 827-834, 2012.

[13] O.M. Kwon, M.J. Park, J.H. Park, S.M. Lee, E.J. Cha, "Stability and stabilization for discrete-time systems with time-varying delays via augmented Lyapunov-Krasovskii functional”, Journal of the Franklin Institute, vol. 350 , no. 3, pp. 521-540, 2013.

[14] L. Hou, and H. Zhu, „Stability of stochastic discrete-time neural networks with discrete delays and the leakage delay" Mathematical Problems in Engineering, vol. 2015, Article ID 306806, 13 pages, 2015.

[15] Y. Li, „Exponential stability results of discrete-time stochastic neural networks with time-varying delays”, Mathematical Problems in Engineering, vol. 2013, Article ID 486257, 10 pages.

[16] R. Saravanakumar, S.B. Stojanovic, D.D. Radosavljevic, C.K. Ahn and H.R. Karimi, „Finite-time passivitybased stability criteria for delayed discrete-time neural networks via new weighted summation inequalities," IEEE Transactions on Neural Networks and Learning Systems, vol. 30, no. 1, pp. 58 - 71, 2019.

[17] D.H. Lin, J. Wu, J.N. Li, ,Less conservative stability condition for uncertain discrete-time recurrent neural networks with time-varying delays," Neurocomputing, vol. 173, pp. 1578-1588, 2016.

[18] M. Wu, F. Liu, P. Shi, Y. He and R. Yokoyama, „Improved free-weighting matrix approach for stability analysis of discrete-time recurrent neural networks with time-varying delay" IEEE Transactions on Circuits and Systems II: Express Briefs, vol. 55, no. 7, pp. 690-694, 2008.

[19] T. Wang, M. Xue, S. Fei, and T. Li, ,Triple Lyapunov functional technique on delay-dependent stability for discrete-time dynamical networks", Neurocomputing, vol. 122, pp. 221-228, 2013

[20] X.-M. Zhang, Q.-L. Han, A. Seuret, and F. Gouaisbaut, ,An improved reciprocally convex inequality and an augmented Lyapunov-Krasovskii functional for stability of linear systems with time-varying delay" Automatica, vol. 84, pp. 221-226, 2017.

[21] O.M. Kwon, M.J. Park, Ju H. Park, S.M. Lee, and E.J. Cha, ,On stability analysis for neural networks with interval time-varying delays via some new augmented Lyapunov-Krasovskii functional” Communications in Nonlinear Science and Numerical Simulation, vol. 19, no. 9, pp 3184-3201, 2014.

[22] M.S. Ali, R. Saravanakumar, and S. Arik, „Novel Ho state estimation of static neural networks with interval time-varying delays via augmented Lyapunov-Krasovskii functional”, Neurocomputing, vol. 171, pp. 949954, 2016.

[23] C.-K. Zhang, Y. He, L. Jiang, W.-J. Lin, and M. Wu, ,Delay-dependent stability analysis of neural networks with time-varying delay: A generalized free-weighting matrix approach", Applied Mathematics and Computation, vol. 294, pp. 102-120, 2017

[24] X.G. Liu, F.X. Wang, and Y.J. Shu, „A novel summation inequality for stability analysis of discrete-time neural networks", Journal of Computational and Applied Mathematics, vol. 304, pp. 160-171, 2016.

[25] Seuret, F. Gouaisbaut, and E. Fridman, ,Stability of discrete-time systems with time-varying delays via a novel summation inequality", IEEE Transactions on Automatic Control, vol. 60, no. 10, pp. 2740-2745, 2015.

[26] C.K. Zhang, Y. He, L. Jiang, and M. Wu, ,An improved summation inequality to discrete-time systems with time-varying delay", Automatica, vol. 74, pp.10-15, 2016.

[27] S. Xiao, L. Xu, H.-B. Zeng, and K.L. Teo, „Improved Stability Criteria for Discrete-time Delay Systems via Novel Summation Inequalities", International Journal of Control, Automation and Systems, vol. 16, no. 4, pp. 1592-1602, 2018

[28] X.-M. Zhang and Q.-L. Han, „New Lyapunov-Krasovskii Functionals for Global Asymptotic Stability of Delayed Neural Networks", IEEE Transactions on Neural Networks, vol. 20, no. 3, pp. 533-539, 2009.

[29] C. Ge, C. Hua, X. Guan, „New delay-dependent stability criteria for neural networks with time-varying delay using delay-decomposition approach”, IEEE Transactions on Neural Networks and Learning Systems, vol. 25 , no. 7 , pp. 1378-1383, 2014 
[30] P. Park, J.W. Ko, and C. Jeong, „Reciprocally convex approach to stability of systems with time-varying delays", Automatica, vol. 47, pp. 235-238, 2011

[31] C.K. Zhang, Y. He, L. Jiang, Q.G. Wang and M. Wu, „Stability analysis of discrete-time neural networks with time-varying delay via an extended reciprocally convex matrix inequality”, IEEE Transactions on Cybernetics, vol. 47, no. 10, pp. 3040-3049, 2017.

[32] D. J. Hill and P. J. Moylan, „Stability results for nonlinear feedback systems”, Automatica, vol. 13, pp. 377382, 1977.

[33] W. Yu, „Passive equivalence of chaos in Lorenz system”, IEEE Transactions on Circuits and Systems I: Fundamental Theory and Applications, vol. 46, no. 7, pp. 876-878, 1999.

[34] C. W. Wu, ,Synchronization in arrays of coupled nonlinear systems: Passivity, circle criterion, and observer design", IEEE Transactions on Circuits and Systems I: Fundamental Theory and Applications, vol. 48, no. 10, pp. 1257-1261, 2001 .

[35] L. Xie, M. Fu, and H. Li, „Passivity analysis and passification for uncertain signal processing systems”, IEEE Transactions on Signal Processing, vol. 46, no. 9, pp. 2394-2403, 1998.

[36] L. O. Chua, „Passivity and complexity,” IEEE Transactions on Circuits and Systems I: Fundamental Theory and Applications, vol. 46, no. 1, pp. 71-82, 1999.

[37] J. Lian and J. Wang, „Passivity of switched recurrent neural networks with time-varying delays”, IEEE Transactions on Neural Networks and Learning Systems, vol. 26, no. 2, pp. 357-366, 2015

[38] J. Zhu, Q. Zhang, and Z. Yuan, „Delay-dependent passivity criterion for discrete-time delayed standard neural network model", Neurocomputing, vol. 73, nos. 7-9, pp. 1384-1393, 2010.

[39] Y. Shu, X. Liu, and Y. Liu, ,Stability and passivity analysis for uncertain discrete-time neural networks with time-varying delay," Neurocomputing, vol. 173, pp. 1706-1714, Jan. 2016.

[40] J. Zhang, L. Ma, and Y. Liu, „Passivity analysis for discretetime neural networks with mixed time-delays and randomly occurring quantization effects", Neurocomputing, vol. 216, pp. 657-665, 2016

[41] Z.-G. Wu, P. Shi, H. Su, and J. Chu, „Passivity analysis for discretetime stochastic Markovian jump neural networks with mixed time delays", IEEE Transactions on Neural Networks, vol. 22, no. 10, pp. 1566-1575, 2011.

[42] D. Zhang and L. Yu, „Passivity analysis for discrete-time switched neural networks with various activation functions and mixed time delays", Nonlinear Dynamics, vol. 67, no. 1, pp. 403-411, 2012.

[43] P. Dorato, „Short-time stability in linear time-varying systems”, DTIC Document, Fort Belvoir, VA, USA, Tech. Rep., 1961.

[44] J. Bai, R. Lu, A. Xue, Q. She, and Z. Shi, „Finite-time stability analysis of discrete-time fuzzy Hopfield neural network", Neurocomputing, vol. 159, pp. 263-267, 2015.

[45] D. L. Debeljkovic, I. M. Buzurovic, S. B. Stojanovic, and A. M. Jovanovic, „Novel conditions for finite time stability of discrete time delay systems" in Proc. International Conference on System Science and Engineering (ICSSE), Budapest, Hungary, Jul. 2013, pp. 177-181.

[46] S. B. Stojanovic, D. L. Debeljkovic, and M. A. Misic, „Finite-time stability for a linear discrete-time delay systems by using discrete convolution: An LMI approach", International Journal of Control, Automation and Systems, vol. 14, no. 4, pp. 1144-1151, 2016.

[47] P. Shi, Y. Zhang, and R. K. Agarwal, ,Stochastic finite-time state estimation for discrete time-delay neural networks with Markovian jumps", Neurocomputing, vol. 151, pp. 168-174, 2015.

[48] K. Mathiyalagan, J. H. Park, and R. Sakthivel, „Novel results on robust finite-time passivity for discrete-time delayed neural networks", Neurocomputing, vol. 177, pp. 585-593, 2016.

[49] L.J. Banu, P. Balasubramaniam, and K. Ratnavelu, „Robust stability analysis for discrete-time uncertain neural networks with leakage time-varying delay", Neurocomputing, vol. 151, pp. 808-816, 2015.

[50] O. M. Kwon, M. J. Park, J. H. Park, S. M. Lee, and E. J. Cha, „New criteria on delay-dependent stability for discrete-time neural networks with time-varying delays", Neurocomputing, vol. 121, pp. 185-194, 2013.

[51] P.T. Nam, P.N. Pathirana, and H. Trinh, „Discrete Wirtinger-based inequality and its application”, Journal of the Franklin Institute, vol. 352, no. 5, pp. 1893-1905, 2015.

[52] X.M. Zhang and Q.-L. Han, ,Abel lemma-based finite-sum inequality and its application to stability analysis for linear discrete time-delay systems", Automatica, vol. 57, pp. 199-202, 2015.

[53] J. Liu, and J. Zhang, „Note on stability of discrete-time time-varying delay systems”, IET Control Theory and Applications, vol. 6, no. 2, pp. 335-339, 2012.

[54] T. Wang, M.X. Xue, C. Zhang, and S.M. Fei, „Improved stability criteria on discrete-time systems with time-varying and distributed delays," International Journal of Automation and Computing, vol. 10, no. 3, pp. 260-266, 2014.

[55] Z. Feng, J. Lam, and G.H. Yang, „Optimal partitioning method for stability analysis of continuous/discrete delay systems,” International Journal of Robust and Nonlinear Control, vol. 25, no. 4, pp. 559-574, 2013. 
[56] S. Stojanovic, M. Stojanovic, M. Stevanovic, „Novel Delay-Dependent Stability Criteria for Discrete-Time Neural Networks with Time-Varying Delay", Mathematical Problems in Engineering, vol. 2018, Article ID 5397870, 15 pages, 2018

[57] L. Jin, Y. Hen, M. Wu, „Improved delay-dependent stability analysis of discrete-time neural networks with time-varying delay", Journal of the Franklin Institute, vol. 354, no. 4, pp. 1922-1936, 2017.

[58] C. Hua, S. Wu, and X. Guan, „New robust stability condition for discrete-time recurrent neural networks with time-varying delays and nonlinear perturbations", Neurocomputing, vol. 219, pp. 203-209, 2017.

[59] X.L. Zhu and G.H. Yang, „Jensen inequality approach to stability analysis of discrete-time systems with time-varying delay," in Proc. American Control Conference, Seattle, WA, USA, 2008, pp. 1644-1649.

[60] Seuret, F. Gouaisbaut, and E. Fridman, ,Stability of systems with fast-varying delay using improved Wirtinger's inequality," in Proc. 52nd IEEE Conference on Decision and Control, Firenze, Italy, 2013, pp. 946-951.

[61] S. Stojanovic, D. Debeljkovic, D. Antic, „The application of different Lyapunov-like functionals and some aggregate norm approximations of the delayed states for finite-time stability analysis of linear discrete timedelay systems", Journal of the Franklin Institute, vol. 351, no. 7, pp. 3914-3931, 2014 